九州大学学術情報リポジトリ

Kyushu University Institutional Repository

\title{
Popular Matchings with Ties and Matroid Constraints
}

Kami yama, Naoyuki

Institute of Mathematics for Industry, Kyushu University | JST, PRESTO

http://hdl. hand le. net/2324/1525809

出版情報 : MI Preprint Series. 2015-6，2015-05-24. 九州大学大学院数理学研究院 バージョン：

権利関係 : 


\section{Preprint Series}

Mathematics for Industry

Kyushu University

\section{Popular Matchings with Ties and Matroid Constraints}

\section{Naoyuki Kamiyama}

\section{2015-6}

( Received May 24, 2015 )

Institute of Mathematics for Industry

Graduate School of Mathematics

Kyushu University

Fukuoka, JAPAN 


\title{
Popular Matchings with Ties and Matroid Constraints
}

\author{
Naoyuki Kamiyama*†
}

\begin{abstract}
In this paper, we consider the popular matching problem with matroid constraints. It is known that if there exists no tie in preference lists of applicants, then this problem can be solved in polynomial time. In this paper, we prove that even if there exist ties in preference lists, this problem can be solved in polynomial time.
\end{abstract}

\section{Introduction}

In this paper, we consider some variation of the popular matching problem. The popular matching problem was originally introduced by Abraham, Irving, Kavitha, and Mehlhorn [1]. In this problem, we are give two disjoint sets of applicants and posts, and each applicant has a preference list over posts in which there may exist ties. A matching $M$ between applicants and posts is said to be popular, if there exists no other matching $N$ such that the number of applicants that prefer $N$ to $M$ is larger than the number of applicants that prefer $M$ to $N$. The concept of popularity was originally proposed by Gärdenfors [10] in the context of matching problems in which agents in both sides have preference lists. The goal of the popular matching problem is to decide whether there exists a popular matching, and find a popular matching if one exists. Abraham, Irving, Kavitha, and Mehlhorn [1] presented polynomial-time algorithms for the popular matching problem with/without ties. Since their seminal paper, several variations of the popular matching problem $[12,19,21,24]$ and related problems $[12,13,14,15,16,20,25,26]$ have been investigated.

In this paper, we consider a matroid generalization of the popular matching problem. More precisely, in our model, capacity constraints for posts are generalized to matroid constraints. In the study of combinatorial optimization, for example, Fleiner [6] generalized capacity constraints in the stable matching problem to matroid constraints, and Zenklusen [27] considered a matroid generalization of the minimum-cost spanning tree problem with degree constraints. It is known [12] that if there exists no tie in preference lists, then this problem can be solved in polynomial time. In this paper, we prove that even if there exist ties in preference lists, we can solve this problem in polynomial time.

Our algorithm is based on the algorithm of Abraham, Irving, Kavitha, and Mehlhorn [1] for the popular matching problem with ties. However, the algorithm of [1] decomposes

\footnotetext{
*Institute of Mathematics for Industry, Kyushu University, Fukuoka, Japan

$\dagger$ JST, PRESTO, Saitama, Japan

${ }^{\ddagger}$ kamiyama@imi.kyushu-u.ac.jp
} 
the vertex set of an input bipartite graph by using Gallai-Edmonds decomposition $[5,8,9]$ (see also [18]) of a bipartite graph based on a maximum-size (one-to-one) matching. Thus, we can not straightforwardly generalize the algorithm of [1] to the matroid setting. Although Manlove and Sng [19] proposed an algorithm for a many-to-one variation of the popular matching with ties, their algorithm copies posts and uses the Gallai-Edmonds decomposition in the one-to-one setting. To overcome this difficulty, we decompose the edge set (instead of the vertex set) of an input bipartite graph bases on a maximum-size common independent set. This decomposition can be regarded as a matroid generalization of the Dulmage-Mendelsohn decomposition [3, 4] of a bipartite graph (see, e.g., [22] for the Dulmage-Mendelsohn type decomposition of the matroid intersection problem).

The rest of this paper is organized as follows. In Section 2, we give the formal definition of our model, and basics of matroids and the matroid intersection problem. In Section 3, we give a characterization of a popular matching in our model. In Section 4, we describe our algorithm, and prove its correctness.

\section{Preliminaries}

For each set $X$ and each element $u$, we define $X+u:=X \cup\{u\}$ and $X-u:=X \backslash\{u\}$. For each sets $X$ and $Y$, we define $X \triangle Y:=(X \backslash Y) \cup(Y \backslash X)$. Assume that we are given a set $X$ and a function $\xi: X \rightarrow \mathbb{Z}$, where $\mathbb{Z}$ is the set of integers. For each subset $Y$ of $X$, we define

$$
\xi(Y):=\sum_{u \in Y} \xi(u)
$$

A pair $\mathbf{M}=(U, \mathcal{I})$ is called a matroid, if $U$ is a finite set and $\mathcal{I}$ is a family of subsets of $U$ satisfying the following conditions.

(I0) $\emptyset \in \mathcal{I}$.

(I1) If $I \in \mathcal{I}$ and $J \subseteq I$, then $J \in \mathcal{I}$.

(I2) If $I, J \in \mathcal{I}$ and $|I|<|J|$, then $I+u \in \mathcal{I}$ for some element $u$ in $J \backslash I$.

We say that $\mathbf{M}$ is a matroid on the ground set $U$.

In this paper, we are given a finite simple (not necessarily complete) bipartite graph $G=(V, E)$. We assume that $V$ is partitioned into two subsets $A$ and $P$, and each edge in $E$ connects a vertex in $A$ and a vertex in $P$. We call a vertex in $A$ an applicant, and a vertex in $P$ a post. For each applicant $a$ in $A$ and each post $p$ in $P$, if there exists an edge in $E$ connecting $a$ and $p$, then we denote by $(a, p)$ this edge. For each vertex $v$ in $V$ and each subset $F$ of $E$, we define $F(v)$ as the set of edges in $F$ incident to $v$.

For each applicant $a$ in $A$, we are given a transitive binary relation $\succsim_{a}$ on $E(a)$ such that at least one of $e \succsim_{a} g$ and $g \succsim_{a} e$ holds for every edges $e, g$ in $E(a)$. Notice that for each applicant $a$ in $A$ and each edges $e, g$ in $E(a)$, there exists a possibility that both $e \succsim_{a} g$ and $g \succsim_{a} e$ hold. For each applicant $a$ in $A$ and each edges $e, g$ in $E(a)$, we use the notation $e \succ_{a} g$, if $e \succsim_{a} g$ and $g \succsim_{a} e$. For each applicant $a$ in $A$ and each edges $e, g$ in $E(a), e \succ_{a} g$ means that $a$ preferes $e$ to $g$. Furthermore, for each applicant $a$ in $A$ and each edges $e, g$ in $E(a)$, if $e \succsim_{a} g$ and $g \succsim_{a} e$ hold, then $a$ is indifferent between $e$ and $g$. 
As in [1], we assume that for each applicant $a$ in $A$, there exists a last resort post $\ell(a)$ in $P$ such that $E(\ell(a))=\{(a, \ell(a))\}$, and $e \succ_{a}(a, \ell(a))$ for every edge $e$ in $E(a)-(a, \ell(a))$. In addition, we assume that for every applicant $a$ in $A$, there exists an edge $e$ in $E(a)$ such that $e \neq(a, \ell(a))$. For each post $p$ in $P$, we are given a matroid $\mathbf{M}_{p}=\left(E(p), \mathcal{I}_{p}\right)$. We assume that for every edge $(a, p)$ in $E$, we have $\{(a, p)\} \in \mathcal{I}_{p}$.

A subset $M$ of $E$ is called a matching in $G$, if

- $|M(a)|=1$ for every applicant $a$ in $A$, and

- $M(p) \in \mathcal{I}_{p}$ for every post $p$ in $P$.

For each subset $F$ of $E$ and and each applicant $a$ in $A$ such that $|F(a)|=1$, we denote by $\mu_{F}(a)$ the unique edge in $F(a)$. For each matchings $M, N$ in $G$, we denote by $\phi(M ; N)$ the number of applicants $a$ in $A$ such that $\mu_{M}(a) \succ_{a} \mu_{N}(a)$. A matching $M$ in $G$ is said to be popular, if for every matching $N$ in $G, \phi(M ; N) \geq \phi(N ; M)$. Then, the goal of the popular matching problem with ties and matroid constraints (PMTM for short) is to decide whether there exists a popular matching in $G$, and find a popular matching if one exists.

\subsection{Basics of matroids}

Let $\mathbf{M}=(U, \mathcal{I})$ be a matroid. A subset of $U$ belonging to $\mathcal{I}$ is called an independent set of $\mathbf{M}$. A subset $C$ of $U$ is called a circuit of $\mathbf{M}$, if $C$ is not an independent set of $\mathbf{M}$, but every proper subset of $C$ is an independent set of $\mathbf{M}$. Assume that we are given an independent set $I$ of $\mathbf{M}$ and an element $u$ in $U \backslash I$ such that $I+u \notin \mathcal{I}$. It is known [23, Proposition 1.1.6] that there exists a unique circuit of $\mathbf{M}$ that is a subset of $I+u$, and $u$ belongs to this unique circuit. This unique circuit is called the fundamental circuit of $u$, and denoted by $\mathrm{C}_{\mathbf{M}}(u, I)$. It is known [23, p.20, Exercise 5] that we have

$$
\mathrm{C}_{\mathbf{M}}(u, I)=\{w \in I+u \mid I+u-w \in \mathcal{I}\} .
$$

Notice that if $\{u\} \in \mathcal{I}$, then $\mathrm{C}_{\mathbf{M}}(u, I)-u \neq \emptyset$.

A maximal independent set of $\mathbf{M}$ is called a base of $\mathbf{M}$. Notice that (I2) implies that every base of $\mathbf{M}$ has the same size. For each subset $X$ of $U$, we define $\mathcal{I} \mid X$ as the family of subsets $I$ of $X$ such that $I \in \mathcal{I}$, and $\mathbf{M} \mid X:=(X, \mathcal{I} \mid X)$. It is known [23, p.20] that for every subset $X$ of $U, \mathbf{M} \mid X$ is a matroid. For each subset $X$ of $U$, we define $\mathbf{r}_{\mathbf{M}}(X)$ as the size of a base of $\mathbf{M} \mid X$. For each disjoint subsets $X, J$ of $U$, we define

$$
\mathrm{p}(J ; X):=\mathrm{r}_{\mathbf{M}}(J \cup X)-\mathrm{r}_{\mathbf{M}}(X) .
$$

For each subset $X$ of $U$, we define

$$
\begin{aligned}
\mathcal{I} / X & :=\{J \subseteq U \backslash X|\mathrm{p}(J ; X)=| J \mid\}, \\
\mathbf{M} / X & :=(U \backslash X, \mathcal{I} / X) .
\end{aligned}
$$

It is known [23, Proposition 3.1.6] that for each subset $X$ of $U, \mathbf{M} / X$ is a matroid.

Lemma 1 (see, e.g., [23, Proposition 3.1.7]). Assume that we are given a matroid $\mathbf{M}=$ $(U, \mathcal{I})$, a subset $X$ of $U$, and a base $B$ of $\mathbf{M} \mid X$. Then, for every element $u$ of $U \backslash X,\{u\}$ is an independent set of $\mathbf{M} / X$ if and only if $B+u$ is an independent set of $\mathbf{M}$. 
Lemma 2 (See, e.g., [23, p.15, Exercise 14]). Assume that we are given a matroid $\mathbf{M}=$ $(U, \mathcal{I})$ and circuits $C_{1}, C_{2}$ of $\mathbf{M}$ such that $C_{1} \cap C_{2} \neq \emptyset$ and $C_{1} \backslash C_{2} \neq \emptyset$. Then, for every element $u$ in $C_{1} \cap C_{2}$ and every element $w$ in $C_{1} \backslash C_{2}$, there exists a circuit $C$ of $\mathbf{M}$ such that $w \in C$ and $C$ is a subset of $\left(C_{1} \cup C_{2}\right)-u$.

Lemma 3. Assume that we are given a matroid $\mathbf{M}=(U, \mathcal{I})$, an independent set $I, J$ of $\mathbf{M}$ such that $J \subseteq I$, and an element $u$ in $U \backslash J$ such that $I+u \notin \mathcal{I}$ and $J+u \notin \mathcal{I}$. Then, $\mathrm{C}_{\mathbf{M}}(u, I)=\mathrm{C}_{\mathbf{M}}(u, J)$.

Proof. Define $C_{1}:=\mathrm{C}_{\mathbf{M}}(u, I)$ and $C_{2}:=\mathrm{C}_{\mathbf{M}}(u, J)$. Assume that $C_{1} \neq C_{2}$. Then, Lemma 2 implies that there exists a circuit $C$ of $\mathbf{M}$ such that $C \subseteq\left(C_{1} \cup C_{2}\right)-u$. Since $C_{1}-u \subseteq I$ and $C_{2}-u \subseteq J \subseteq I$, we have $C \subseteq I$. This contradicts the fact that $I \in \mathcal{I}$.

Lemma 4. Assume that we are given a matroid $\mathbf{M}=(U, \mathcal{I})$, an independent set $I$ of $\mathbf{M}$, and a subset $J$ of $U \backslash I$ such that $I \cup J \in \mathcal{I}$. Furthermore, we assume that we are given subsets $X, Y$ of $U \backslash J$ such that $(I \cup X) \backslash Y \in \mathcal{I}$ and $I+x \notin \mathcal{I}$ for every element $x$ in $X$. Then, $((I \cup X) \backslash Y) \cup J$ is an independent set of $\mathbf{M}$.

Proof. We prove this lemma by contradiction. Assume that $((I \cup X) \backslash Y) \cup J \notin \mathcal{I}$. This implies that there exists a circuit $C$ of $\mathbf{M}$ such that $C \subseteq((I \cup X) \backslash Y) \cup J$. If $C \cap J=\emptyset$, then this contradicts the fact that $(I \cup X) \backslash Y$ is an independent set of $\mathbf{M}$. Thus, there exists an element $u$ in $J \cap C$. If $C \cap X=\emptyset$, then this contradicts the fact that $I \cup J$ is an independent set of $\mathbf{M}$. Thus, $X \cap C$ is not empty. Assume that $X \cap C=\left\{x_{1}, x_{2}, \ldots, x_{k}\right\}$. Since $u \in J, J \cap X=\emptyset$, and $J \cap I=\emptyset$, we have $u \notin \mathbf{C}_{\mathbf{M}}\left(x_{i}, I\right)$ for every $i=1,2, \ldots, k$.

Here we consider the following procedure.

Step 1. Set $i:=1$ and $C_{0}:=C$.

Step 2. If $i \leq k$, then do the following steps.

(2-a) If $x_{i} \notin C_{i-1}$, then set $C_{i}:=C_{i-1}$ and go to (2-c).

(2-b) Find a circuit $C_{i}$ of $\mathbf{M}$ such that $u \in C_{i}$ and $C_{i} \subseteq\left(C_{i-1} \cup \mathrm{C}_{\mathbf{M}}\left(x_{i}, I\right)\right)-x_{i}$.

(2-c) Update $i:=i+1$ and go back to the beginning of Step 2.

Step 3. Output $C_{k}$.

In Step (2-b), since $x_{i} \in C_{i-1} \cap \mathrm{C}_{\mathbf{M}}\left(x_{i}, I\right)$ and $u \in C_{i-1} \backslash \mathrm{C}_{\mathbf{M}}\left(x_{i}, I\right)$, Lemma 2 implies that there exists a circuit $C_{i}$ of $\mathbf{M}$ such that $u \in C_{i}$ and $C_{i} \subseteq\left(C_{i-1} \cup \mathrm{C}_{\mathbf{M}}\left(x_{i}, I\right)\right)-x_{i}$. It is not difficult to see that $C_{k}$ is a subset of $I \cup J$, which contradicts the fact that $I \cup J \in \mathcal{I}$. This completes the proof.

Assume that we are given $k$ matroids $\mathbf{M}_{1}=\left(U_{1}, \mathcal{I}_{1}\right), \mathbf{M}_{2}=\left(U_{2}, \mathcal{I}_{2}\right), \ldots, \mathbf{M}_{k}=\left(U_{k}, \mathcal{I}_{k}\right)$ such that $U_{i} \cap U_{j}=\emptyset$ for every distinct $i, j=1,2, \ldots, k$. Define

$$
\bigoplus_{i=1}^{k} \mathcal{I}_{i}:=\left\{X \subseteq \bigcup_{i=1}^{k} U_{i} \mid X \cap U_{i} \text { is an independent set of } \mathbf{M}_{i} \text { for every } i=1,2, \ldots, k\right\} \text {. }
$$


Furthermore, we define

$$
\bigoplus_{i=1}^{k} \mathbf{M}_{i}:=\left(\bigcup_{i=1}^{k} U_{i}, \bigoplus_{i=1}^{k} \mathcal{I}_{i}\right) .
$$

It is not difficult to see that $\bigoplus_{i=1}^{k} \mathbf{M}_{i}$ is a matroid.

Let $\mathbf{M}=(U, \mathcal{I})$ and $\mathbf{N}=(U, \mathcal{J})$ be matroids. A subset $I$ of $U$ is called a common independent set of $\mathbf{M}$ and $\mathbf{N}$, if $I \in \mathcal{I} \cap \mathcal{J}$. We denote by $\mathcal{B}_{\mathbf{M N}}$ and $\gamma_{\mathbf{M N}}$ the family and the size of maximum-size common independent sets of $\mathbf{M}$ and $\mathbf{N}$, respectively. We can find a maximum-size common independent set of $\mathbf{M}$ and $\mathbf{N}$ in $O\left(|U|^{2.5} \mathrm{EO}\right)$ by using the algorithm of Cunningham [2], where EO is the time required to decide whether $X+u$ is an independent set of $\mathbf{L}$ for every matroid $\mathbf{L}$ in $\{\mathbf{M}, \mathbf{N}\}$, every common independent set $X$ of $\mathbf{M}$ and $\mathbf{N}$, and every element $u$ in $U \backslash X$. Furthermore, for every function $\xi: U \rightarrow \mathbb{Z}$, we can find a maximum-size common independent set $I$ of $\mathbf{M}$ and $\mathbf{N}$ such that

$$
\xi(I)=\max \left\{\xi(J) \mid J \in \mathcal{B}_{\mathrm{MN}}\right\}
$$

in $O\left(|U|^{3} \mathrm{EO}\right)$ time by using the algorithm of Frank [7].

\subsection{Auxiliary graphs and decomposition}

Assume that we are given matroids $\mathbf{M}=(U, \mathcal{I})$ and $\mathbf{N}=(U, \mathcal{J})$, and a common independent set $I$ of $\mathbf{M}$ and $\mathbf{N}$. Then, we define a directed graph $\mathbf{D}_{\mathbf{M N}}(I)$ as follows. The vertex set of $\mathrm{D}_{\mathrm{MN}}(I)$ is $U$. For each elements $u$ of $U \backslash I$ and $v$ of $I$,

- there exists an arc from $v$ to $u$ in $\mathrm{D}_{\mathbf{M N}}(I)$ if and only if $I+u \notin \mathcal{I}$ and $v \in \mathbf{C}_{\mathbf{M}}(u, I)$,

- there exists an arc from $u$ to $v$ in $\mathrm{D}_{\mathbf{M N}}(I)$ if and only if $I+u \notin \mathcal{J}$ and $v \in \mathbf{C}_{\mathbf{N}}(u, I)$.

These are all arcs of $\mathrm{D}_{\mathrm{MN}}(I)$. Define

$$
\begin{aligned}
& \mathrm{T}_{\mathbf{M}}(I):=\{u \in U \backslash I \mid I+u \in \mathcal{I}\}, \\
& \mathrm{T}_{\mathbf{N}}(I):=\{u \in U \backslash I \mid I+u \in \mathcal{J}\} .
\end{aligned}
$$

Lemma 5 (see, e.g., [17, Lemma 13.30]). Assume that we are given matroids $\mathbf{M}$ and $\mathbf{N}$ on the same ground set $U$ and a common independent set $I$ of $\mathbf{M}$ and $\mathbf{N}$. Then, $I$ is a maximum-size common independent set of $\mathbf{M}$ and $\mathbf{N}$ if and only if there exists no directed path in $\mathrm{D}_{\mathbf{M N}}(I)$ from a vertex in $\mathrm{T}_{\mathbf{M}}(I)$ to a vertex in $\mathrm{T}_{\mathbf{N}}(I)$.

Assume that $I$ is a maximum-size common independent set of $\mathbf{M}$ and $\mathbf{N}$. We denote by $\Omega_{\mathbf{M N}}^{+}(I)$ the set of elements $u$ in $U$ such that there exists a directed path in $\mathrm{D}_{\mathbf{M N}}(I)$ from a vertex in $\mathrm{T}_{\mathbf{M}}(I)$ to $u$. In addition, we define $\Omega_{\mathbf{M N}}^{-}(I)$ as the set of elements $u$ in $U$ such that there exists a directed path in $\mathrm{D}_{\mathbf{M N}}(I)$ from $u$ to a vertex in $\mathrm{T}_{\mathbf{N}}(I)$. Notice that since $I$ is a maximum-size common independent set of $\mathbf{M}$ and $\mathbf{N}$, Lemma 5 implies that $\Omega_{\mathbf{M N}}^{+}(I)$ and $\Omega_{\mathbf{M N}}^{-}(I)$ are disjoint. Define

$$
\Omega_{\mathrm{MN}}(I):=U \backslash\left(\Omega_{\mathrm{MN}}^{+}(I) \cup \Omega_{\mathrm{MN}}^{-}(I)\right) .
$$


Lemma 6. Assume that we are given matroids $\mathbf{M}$ and $\mathbf{N}$ on the same ground set $U$ and a common independent set $I$ of $\mathbf{M}$ and $\mathbf{N}$. Furthermore, we assume that we are given a vertex $u$ in $\mathrm{T}_{\mathbf{M}}(I) \cup I$ and a vertex $v$ in $\mathrm{T}_{\mathbf{N}}(I) \cup I$, and a shortest directed path $L$ in $\mathrm{D}_{\mathrm{MN}}(I)$ from $u$ to $v$. Then, $I \triangle K$ is a common independent set of $\mathbf{M}$ and $\mathbf{N}$, where $K$ is the set of elements in $U$ that $L$ goes through (notice that since $L$ is a shorted directed path, $L$ goes through each vertex at most once).

Proof. This lemma immediately follows from [17, Lemma 13.27] and Lemma 4.

Lemma 7 (see, e.g., [11, Lemma 4.2]). Assume that we are given matroids $\mathbf{M}$ and $\mathbf{N}$ on the same ground set. Then, for every maximum-size common independent sets $I, J$ of $\mathbf{M}$ and $\mathbf{N}$,

$$
\begin{aligned}
& J \cap \Omega_{\mathbf{M N}}^{-}(I) \text { is a base of } \mathbf{M} \mid \Omega_{\mathbf{M N}}^{-}(I), \\
& J \cap\left(\Omega_{\mathbf{M N}}(I) \cup \Omega_{\mathbf{M N}}^{-}(I)\right) \text { is a base of } \mathbf{M} \mid\left(\Omega_{\mathbf{M N}}(I) \cup \Omega_{\mathbf{M N}}^{-}(I)\right), \\
& J \cap \Omega_{\mathbf{M N}}^{+}(I) \text { is a base of } \mathbf{N} \mid \Omega_{\mathbf{M N}}^{+}(I), \\
& J \cap\left(\Omega_{\mathbf{M N}}^{+}(I) \cup \Omega_{\mathbf{M N}}(I)\right) \text { is a base of } \mathbf{N} \mid\left(\Omega_{\mathbf{M N}}^{+}(I) \cup \Omega_{\mathbf{M N}}(I)\right) .
\end{aligned}
$$

Although the following lemma is well-known, we give its proof for completeness.

Lemma 8. Assume that we are given matroids $\mathbf{M}=(U, \mathcal{I})$ and $\mathbf{N}=(U, \mathcal{J})$. Then, for every maximum-size common independent sets $I, J$ of $\mathbf{M}$ and $\mathbf{N}$, we have

$$
\begin{aligned}
& \Omega_{\mathbf{M N}}^{+}(I)=\Omega_{\mathbf{M N}}^{+}(J), \\
& \Omega_{\mathbf{M N}}(I)=\Omega_{\mathbf{M N}}(J), \\
& \Omega_{\mathbf{M N}}^{-}(I)=\Omega_{\mathbf{M N}}^{-}(J) .
\end{aligned}
$$

Proof. For simplicity, we define $\mathrm{D}_{\mathbf{M N}}(\cdot):=\mathrm{D}(\cdot), \Omega_{\mathbf{M N}}^{+}(\cdot):=\Omega^{+}(\cdot)$, and $\Omega_{\mathbf{M N}}^{-}(\cdot):=\Omega^{-}(\cdot)$. Let $I, J$ be maximum-size common independent sets of $\mathbf{M}$ and $\mathbf{N}$.

We first prove that $\Omega^{+}(I)=\Omega^{+}(J)$. For proving this, we first prove that $\Omega^{+}(J) \subseteq$ $\Omega^{+}(I)$. Assume that $\mathrm{T}_{\mathbf{M}}(J) \nsubseteq \Omega^{+}(I)$, and let $u$ be an element in $\mathrm{T}_{\mathbf{M}}(J) \backslash \Omega^{+}(I)$. Then, $J+u \in \mathcal{I}$ and (I1) imply that

$$
(J+u) \cap\left(\Omega(I) \cup \Omega^{-}(I)\right)=\left(J \cap\left(\Omega(I) \cup \Omega^{-}(I)\right)\right)+u \in \mathcal{I},
$$

which contradicts (2). Assume that there exists an arc in $\mathrm{D}(J)$ from a vertex $u$ in $\Omega^{+}(I)$ to a vertex $v$ in $\Omega(I) \cup \Omega^{-}(I)$. If $u$ is in $I$, then $J+v-u \in \mathcal{I}$. This and (I1) imply that

$$
(J+v-u) \cap\left(\Omega(I) \cup \Omega^{-}(I)\right)=\left(J \cap\left(\Omega(I) \cup \Omega^{-}(I)\right)\right)+v \in \mathcal{I} .
$$

This contradicts (2). If $u$ is not in $I$, then $J+u-v \in \mathcal{J}$. This and (I1) imply that

$$
(J+u-v) \cap \Omega^{+}(I)=\left(J \cap \Omega^{+}(I)\right)+u \in \mathcal{J} .
$$

This contradicts (3). By exchanging the roles of $I$ and $J$, we can prove that $\Omega^{+}(I) \subseteq$ $\Omega^{+}(J)$, which implies that $\Omega^{+}(J)=\Omega^{+}(I)$. 
Next we prove that $\Omega^{-}(J)=\Omega^{-}(I)$. For proving this, we first prove that $\Omega^{-}(J) \subseteq$ $\Omega^{-}(I)$. Assume that $\mathrm{T}_{\mathbf{N}}(J) \nsubseteq \Omega^{-}(I)$, and let $u$ be an element in $\mathrm{T}_{\mathbf{N}}(J) \backslash \Omega^{-}(I)$. Then, $J+u \in \mathcal{J}$ and (I1) imply that

$$
(J+u) \cap\left(\Omega^{+}(I) \cup \Omega(I)\right)=\left(J \cap\left(\Omega^{+}(I) \cup \Omega(I)\right)\right)+u \in \mathcal{J} .
$$

This contradicts (4). Assume that there exists an arc in $\mathrm{D}(J)$ from $u$ in $\Omega^{+}(I) \cup \Omega(I)$ to $v$ in $\Omega^{-}(I)$. If $u$ is in $I$, then $J+v-u \in \mathcal{I}$. This and (I1) imply that

$$
(J+v-u) \cap \Omega^{-}(I)=\left(J \cap \Omega^{-}(I)\right)+v \in \mathcal{I} .
$$

This contradicts (1). If $u$ is not in $I$, then $J+u-v \in \mathcal{J}$. This and (I1) imply that

$$
(J+u-v) \cap\left(\Omega^{+}(I) \cup \Omega(I)\right)=\left(J \cap\left(\Omega^{+}(I) \cup \Omega(I)\right)\right)+u \in \mathcal{J} .
$$

This contradicts (4). By exchanging the roles of $I$ and $J$, we can prove that $\Omega^{-}(I) \subseteq$ $\Omega^{-}(J)$, which implies that $\Omega^{-}(J)=\Omega^{-}(I)$. This completes the proof.

Lemma 8 implies that for every matroids $\mathbf{M}$ and $\mathbf{N}$ on the same ground set, $\Omega_{\mathbf{M N}}^{+}(I)$, $\Omega_{\mathrm{MN}}(I)$, and $\Omega_{\mathrm{MN}}^{-}(I)$ do not depend a choice of a maximum-size common independent set $I$ of $\mathbf{M}$ and $\mathbf{N}$. Thus, for each matroids $\mathbf{M}$ and $\mathbf{N}$ on the same ground set and some maximum-size common independent set $I$, we define

$$
\begin{aligned}
& \Omega_{\mathrm{MN}}^{+}:=\Omega_{\mathrm{MN}}^{+}(I), \\
& \Omega_{\mathrm{MN}}:=\Omega_{\mathrm{MN}}(I), \\
& \Omega_{\mathrm{MN}}^{-}:=\Omega_{\mathrm{MN}}^{-}(I),
\end{aligned}
$$

respectively.

\section{Characterization}

In this section, we give a characterization of a popular matching. Define

$$
\mathcal{U}:=\{F \subseteq E|| F(a) \mid \leq 1 \text { for every applicant } a \text { in } A\},
$$

and $\mathcal{A}:=(E, \mathcal{U})$. It is not difficult to see that $\mathcal{A}$ is a matroid. Define

$$
\mathbf{P}:=\bigoplus_{p \in P} \mathbf{M}_{p}
$$

Notice that for each subset $M$ of $E, M$ is a matching in $G$ if and only if $M$ is a common independent set of $\mathbf{A}$ and $\mathbf{P}$ such that $|M|=|A|$. For each applicant $a$ in $A$, we define $f(a)$ by

$$
f(a):=\left\{e \in E(a) \mid e \succsim_{a} g \text { for every edge } g \text { in } E(a)\right\} .
$$


Notice that for each applicant $a$ in $A$, there exists a possibility that $|f(a)|>1$. Define

$$
\begin{aligned}
\Gamma & :=\bigcup_{a \in A} f(a), \\
\mathbf{B} & :=\mathbf{A} \mid \Gamma, \\
\mathbf{Q} & :=\mathbf{P} \mid \Gamma \quad\left(=\bigoplus_{p \in P}\left(\mathbf{M}_{p} \mid \Gamma(p)\right)\right) .
\end{aligned}
$$

In addition, we define

$$
T:=\left\{e \in E \backslash \Gamma \mid\{e\} \text { is an independent set of } \mathbf{P} /\left(\Omega_{\mathbf{B Q}}^{+} \cup \Omega_{\mathbf{B Q}}\right)\right\} .
$$

Notice that for each applicant $a$ in $A$, since there exists the last resort post $\ell(a), T(a) \neq \emptyset$. For each applicant $a$ in $A$, we define $s(a)$ by

$$
s(a):=\left\{e \in T(a) \mid e \succsim_{a} g \text { for every edge } g \text { in } T(a)\right\} .
$$

In addition, we define

$$
\begin{aligned}
& S:=\bigcup_{a \in A} s(a), \\
& \Pi:=\Gamma \cup S .
\end{aligned}
$$

The following characterization can be regarded as a generalization of [1, Lemma 3.5].

Theorem 9. For each matching $M$ in $G, M$ is a popular matching in $G$ if and only if

(P1) $M \cap \Gamma$ is a maximum-size common independent set of $\mathbf{B}$ and $\mathbf{Q}$, and

(P2) $M$ is a subset of $\Pi$.

We first give lemmas that are necessary for proving the only if-part of Theorem 9.

Lemma 10. Assume that we are given a popular matching $M$ in $G$. Then, $M \cap \Gamma$ is a maximum-size common independent set of $\mathbf{B}$ and $\mathbf{Q}$.

Since the proof of Lemma 10 is long, we leave it to Section 3.1.

Lemma 11. Assume that we are given a popular matching $M$ in $G$. Then, there exists no applicant $a$ in $A$ such that $e \succ_{a} \mu_{M}(a) \succ_{a} g$ for edges e in $f(a)$ and $g$ in $s(a)$.

Proof. For proving this lemma by contradiction, we assume that there exists an applicant $a$ in $A$ such that $e \succ_{a} \mu_{M}(a) \succ_{a} g$ for edges $e$ in $f(a)$ and $g$ in $s(a)$. Since $M$ is a popular matching in $G$, Lemma 10 implies that $M \cap \Gamma$ is a maximum common independent set of $\mathbf{B}$ and $\mathbf{Q}$. This and Lemma 7 imply that $M \cap\left(\Omega_{\mathbf{B Q}}^{+} \cup \Omega_{\mathbf{B Q}}\right)$ is a base of $\mathbf{Q} \mid\left(\Omega_{\mathbf{B Q}}^{+} \cup \Omega_{\mathbf{B Q}}\right)$ $\left(=\mathbf{P} \mid\left(\Omega_{\mathbf{B Q}}^{+} \cup \Omega_{\mathbf{B Q}}\right)\right)$. Define

$$
N:=\left(M \cap\left(\Omega_{\mathbf{B Q}}^{+} \cup \Omega_{\mathbf{B Q}}\right)\right)+\mu_{M}(a) .
$$

Since $N \subseteq M$ and $M$ is an independent set of $\mathbf{P}, N$ is an independent set of $\mathbf{P}$. Thus, (5) and Lemma 1 imply that $\mu_{M}(a)$ is an independent set of $\mathbf{P} /\left(\Omega_{\mathbf{B Q}}^{+} \cup \Omega_{\mathbf{B Q}}\right)$. However, this contradicts that fact that $\mu_{M}(a) \succ_{a} g$ for an edge $g$ in $s(a)$. This completes the proof. 
Lemma 12. Assume that we are given a popular matching $M$ in $G$. Then, there exists no applicant $a$ in $A$ such that $\mu_{M}(a) \notin \Pi$.

We leave the proof of Lemma 12 to Section 3.2.

Next we give a lemma that is necessary for proving the if-part of Theorem 9. For each matching $M$ in $G$, we denote by $\mathrm{b}(M)$ the set of edges $e=(a, p)$ in $E$ such that $e \succ_{a} g$ for an edge $g$ in $s(a)$.

Lemma 13. For every matching $M$ in $G$, we have $\gamma_{\mathbf{B Q}} \geq|\mathrm{b}(M)|$.

Proof. For proving this lemma by contradiction, we assume that there exists a matching $M$ in $G$ such that $\gamma_{\mathbf{B Q}}<|\mathrm{b}(M)|$. Let $N$ be a maximum-size common independent set in $\mathbf{B}$ and $\mathbf{Q}$ such that

$$
\forall N^{\prime} \in \mathcal{B}_{\mathrm{BQ}}:|M \cap N| \geq\left|M \cap N^{\prime}\right|
$$

Since $\gamma_{\mathbf{B Q}}<|\mathrm{b}(M)|$, we have $|N|<|\mathrm{b}(M)|$. In addition, since $M$ is an independent set of $\mathbf{P}$, (I1) implies that $\mathrm{b}(M)$ is an independent set of $\mathbf{P}$. Furthermore, $N$ is an independent set of $\mathbf{P}$. Thus, (I2) implies that there exists an edge $e=(a, p)$ in $\mathrm{b}(M) \backslash N$ such that $N+e$ is an independent set of $\mathbf{P}$. Notice that since $e$ is in $\mathrm{b}(M), e \succ_{a} g$ for an edge $g$ in $s(a)$.

We first consider the case where $e \notin \Gamma$. Since $N+e$ is an independent set of $\mathbf{P}$, (I1) implies that

$$
\left(N \cap\left(\Omega_{\mathbf{B Q}}^{+} \cup \Omega_{\mathbf{B Q}}\right)\right)+e
$$

is an independent set of $\mathbf{P}$. Since it follows from Lemma 7 that $N \cap\left(\Omega_{\mathbf{B Q}}^{+} \cup \Omega_{\mathbf{B Q}}\right)$ is a base of $\mathbf{Q} \mid\left(\Omega_{\mathbf{B Q}}^{+} \cup \Omega_{\mathbf{B Q}}\right)\left(=\mathbf{P} \mid\left(\Omega_{\mathbf{B Q}}^{+} \cup \Omega_{\mathbf{B Q}}\right)\right),\{e\}$ is an independent set of $\mathbf{P} /\left(\Omega_{\mathbf{B Q}}^{+} \cup \Omega_{\mathbf{B Q}}\right)$. This contradicts the fact that $e \succ_{a} g$ for an edge $g$ in $s(a)$.

Next we consider the case where $e \in \Gamma$, i.e., $N+e \subseteq \Gamma$. Since $N$ is a maximum-size common independent set in $\mathbf{B}$ and $\mathbf{Q}, N+e$ is not an independent set of $\mathbf{A}$. That is, $|N(a)|=1$. In addition, since $e \in M$ and $M$ is a matching in $G, \mu_{N}(a) \notin M$. Thus,

$$
\left|\left(N+e-\mu_{N}(a)\right) \cap M\right|=|M \cap N|+1 .
$$

Since $N$ is an independent set of $\mathbf{B}, N+e-\mu_{N}(a)$ is an independent set of $\mathbf{B}$. Since $N+e$ is an independent set of $\mathbf{Q}$, (I1) implies that $N+e-\mu_{N}(a)$ is a maximum-size common independent set in $\mathbf{B}$ and $\mathbf{Q}$. These and (7) contradict (6), which completes the proof.

We are now ready to prove Theorem 9 .

Proof of Theorem 9. Since the only if-part follows from Lemmas 10 and 12, we prove the if-part. Let $M$ be a matching in $G$ satisfying (P1) and (P2), and assume that we are given a matching $N$ in $G$. We denote by $A_{M}$ and $A_{N}$ be the sets of applicants $a$ in $A$ such that $\mu_{M}(a) \succ_{a} \mu_{N}(a)$ and $\mu_{N}(a) \succ_{a} \mu_{M}(a)$, respectively. If we can prove that $\left|A_{M}\right| \geq\left|A_{N}\right|$, then the proof is done. For proving this, it is sufficient to construct an injective function $\tau: A_{N} \rightarrow A_{M}$.

Lemma 13 and (P1) imply that there exists an injective function

$$
\varphi: \mathrm{b}(N) \backslash(M \cap \Gamma) \rightarrow(M \cap \Gamma) \backslash \mathrm{b}(N) .
$$

Let $a$ be an applicant in $A_{N}$. Since $\mu_{N}(a) \succ_{a} \mu_{M}(a)$, (P2) implies that $\mu_{M}(a)$ is in $s(a)$. Thus, $\mu_{N}(a)$ is in $\mathrm{b}(N) \backslash(M \cap \Gamma)$. Here we consider the following procedure. 
Step 1. Set $i:=1$, and define $e_{1}=\left(a_{1}, p_{1}\right)$ as $\varphi\left(\mu_{N}(a)\right)$.

Step 2. If we have $e_{i} \succ_{a_{i}} \mu_{N}\left(a_{i}\right)$, then we define $\tau(a):=a_{i}$ and halt. Otherwise, define $e_{i+1}:=\left(a_{i+1}, p_{i+1}\right)$ as $\varphi\left(\mu_{N}\left(a_{i}\right)\right)$ and update $i:=i+1$. Then, repeat Step 2.

For proving that this procedure is well-defined, it is sufficient to prove in Step 2, $\mu_{N}\left(a_{i}\right)$ is in $\mathrm{b}(N) \backslash(M \cap \Gamma)$. Since $e_{i} \in(M \cap \Gamma) \backslash \mathrm{b}(N)$, if $e_{i} \in N$, then $e_{i} \in f\left(a_{i}\right)$ implies that $e_{i} \in \mathrm{b}(N)$. This contradicts the fact that $e_{i} \in(M \cap \Gamma) \backslash \mathrm{b}(N)$. Thus, $e_{i} \notin N$. In addition, since $e_{i} \in f\left(a_{i}\right)$, if $e_{i} \nsucc_{a_{i}} \mu_{N}\left(a_{i}\right)$, then $\mu_{N}\left(a_{i}\right) \in f\left(a_{i}\right)$. Thus, $\mu_{N}\left(a_{i}\right) \in \mathrm{b}(N) \backslash(M \cap \Gamma)$.

Since $G$ is finite and $\varphi$ is injective, this procedure halts. Thus, since $\varphi$ is injective, $\tau$ is clearly injective. This completes the proof.

\subsection{Proof of Lemma 10}

Here we give a proof of Lemma 10. In the sequel, for each common independent set $I$ of $\mathbf{B}$ and $\mathbf{Q}$, we do not distinguish between each vertex of $\mathbf{D}_{\mathbf{B Q}}(I)$ and the edge in $\Gamma$ that it corresponds to. That is, we may call a vertex of $\mathrm{D}_{\mathbf{B Q}}(I)$ an edge. Since $\mathrm{D}_{\mathbf{B Q}}(I)$ is a directed graph (i.e., it contains only arcs), it does not make any confusion.

Since $M$ is a matching in $G, M$ is a common independent set of $\mathbf{A}$ and $\mathbf{P}$. This and (I1) imply that $M \cap \Gamma$ is a common independent set of $\mathbf{B}$ and $\mathbf{Q}$. For proving this lemma by contradiction, we assume that $M \cap \Gamma$ is not a maximum-size common independent set of $\mathbf{B}$ and $\mathbf{Q}$. It follows from this and Lemma 5 that there exist an edge $g=(b, q)$ in $\mathrm{T}_{\mathbf{B}}(M \cap \Gamma)$ and an edge $h=(c, r)$ in $\mathrm{T}_{\mathbf{Q}}(M \cap \Gamma)$ such that there exists a directed path in $\mathrm{D}_{\mathbf{B Q}}(M \cap \Gamma)$ from $g$ to $h$. Let $L$ be a shortest directed path in $\mathrm{D}_{\mathbf{B Q}}(M \cap \Gamma)$ from $g$ to $h$, and we denote by $K=\left\{e_{1}, e_{2}, \ldots, e_{k}\right\}$ the set of edges in $\Gamma$ (i.e., vertices of $\mathbf{D}_{\mathbf{B Q}}(M \cap \Gamma)$ ) that $L$ goes through. Furthermore, we assume that $e_{i}:=\left(a_{i}, p_{i}\right)$ for each $i=1,2, \ldots, k$, and $L$ goes through $e_{1}, e_{2}, \ldots, e_{k}$ in this order. Notice that $e_{1}=g, e_{k}=h, k$ is odd, and $e_{i} \in M$ for every $i=2,4, \ldots, k-1$. Define $N:=M \triangle K$ and $N_{0}:=N-\mu_{M}(b)$. Lemma 6 implies that $N \cap \Gamma\left(=N_{0} \cap \Gamma\right)$ is an independent set of $\mathbf{Q}$.

For completing a proof of Lemma 10, we first prove necessary lemmas (Section 3.1.1), and then complete a proof of Lemma 3.1 (Section 3.1.2).

\subsubsection{Necessary lemmas}

Here we prove necessary lemmas for proving Lemma 10.

Lemma 14. For every post $p$ in $P-r$, we have $N(p) \in \mathcal{I}_{p}$ and $N_{0}(p) \in \mathcal{I}_{p}$.

Proof. Let $p$ be a post in $P-r$. By using Lemma 4, we prove that $N(p) \in \mathcal{I}_{p}$. If we can prove this, then (I1) implies that $N_{0}(p) \in \mathcal{I}_{p}$.

Define $I:=M(p) \cap \Gamma, J:=M(p) \backslash \Gamma, X:=K(p) \backslash M$, and $Y:=M(p) \cap K$. Then,

$$
\begin{aligned}
(I \cup X) \backslash Y & =N(p) \cap \Gamma, \\
((I \cup X) \backslash Y) \cup J & =N(p) .
\end{aligned}
$$

Since $M$ is an independent set of $\mathbf{P}$, the definition of $\mathbf{P}$ implies that $I \cup J \in \mathcal{I}_{r}$. In addition, the definition of $\mathbf{Q}$ implies that $(I \cup X) \backslash Y$ is an independent set of $\mathbf{M}_{p} \mid \Gamma(p)$, 
i.e., $\mathbf{M}_{p}$. Furthermore, for every edge $e$ in $X$, it follows from the definition of $\mathrm{D}_{\mathbf{B Q}}(M \cap \Gamma)$ that $(M \cap \Gamma)+e$ is not an independent set of $\mathbf{Q}$. Thus, since $M \cap \Gamma$ is an independent set of $\mathbf{Q}$, the definition of $\mathbf{Q}$ implies that $I+e$ is not an independent set of $\mathbf{M}_{p} \mid \Gamma(p)$, i.e., $\mathbf{M}_{p}$. Thus, Lemma 4 implies that $N(p) \in \mathcal{I}_{p}$.

Lemma 15. For every applicant $a$ in $A-b$, (i) $|N(a)|=1$, and (ii) $\mu_{N}(a) \succsim_{a} \mu_{M}(a)$.

Proof. For every $i=2,4, \ldots, k-1$, since there exists an arc in $\mathrm{D}_{\mathbf{B Q}}(M \cap \Gamma)$ from $e_{i}$ to $e_{i+1}, M+e_{i+1}-e_{i}$ is an independent set of $\mathbf{B}$. Thus, the definitions of $\mathbf{A}$ implies that we have $a_{i}=a_{i+1}$ for every $i=2,4, \ldots, k-1$. This implies that for every applicant $a$ in $A-b$, since $|M(a)|=1$, we have $|N(a)|=1$. Furthermore, since $K \subseteq \Gamma, \mu_{N}(a) \in f(a)$ for every applicant $a$ in $A-b$, which implies (ii). This completes the proof.

Lemma 16. (i) $g \succ_{b} \mu_{M}(b)$, and (ii) $\left|N_{0}(b)\right|=1$.

Proof. Since $g \in \mathrm{T}_{\mathbf{B}}(M \cap \Gamma), M(b) \cap \Gamma=\emptyset$. Thus, $\mu_{M}(b) \notin \Gamma$. This and $g \in \Gamma$ imply that $g \succ_{b} \mu_{M}(b)$. As proved in the proof of Lemma 15, $a_{i}=a_{i+1}$ for every $i=2,4, \ldots, k-1$. Thus, since $\mu_{M}(b) \notin \Gamma$, we have $K(b)=\{g\}$. This and $M(b)=\left\{\mu_{M}(b)\right\}$ imply (ii).

We denote by $L^{\prime}$ the subpath of $L$ from $g$ to $e_{k-1}$. Since $L$ is a shortest directed path in $\mathrm{D}_{\mathbf{B Q}}(M \cap \Gamma)$ from $g$ to $h, L^{\prime}$ is a shortest directed path in $\mathrm{D}_{\mathbf{B Q}}(M \cap \Gamma)$ from $g$ to $e_{k-1}$. Define $K^{\prime}:=K-h$. Then, $N-h=M \triangle K^{\prime}$. Lemma 6 implies that $(N-h) \cap \Gamma$ is an independent set of $\mathbf{Q}$.

Lemma 17. $N(r)-h \in \mathcal{I}_{r}$ and $N_{0}(r)-h \in \mathcal{I}_{r}$.

Proof. By using Lemma 4, we prove that $N(r)-h \in \mathcal{I}_{r}$. If we can prove this, then (I1) implies that $N_{0}(r)-h \in \mathcal{I}_{r}$.

Define $I:=M(r) \cap \Gamma, J:=M(r) \backslash \Gamma, X:=K^{\prime}(r) \backslash M$, and $Y:=M(r) \cap K^{\prime}$. Then,

$$
\begin{aligned}
(I \cup X) \backslash Y & =(N(r)-h) \cap \Gamma, \\
((I \cup X) \backslash Y) \cup J & =N(r)-h .
\end{aligned}
$$

Since $M$ is an independent set of $\mathbf{P}$, the definition of $\mathbf{P}$ implies that $I \cup J$ is in $\mathcal{I}_{r}$. In addition, the definition of $\mathbf{Q}$ implies that implies that $(I \cup X) \backslash Y$ is in $\mathcal{I}_{r}$. For every edge $e$ in $X$, the definition of $\mathrm{D}_{\mathbf{B Q}}(M \cap \Gamma)$ implies that $(M \cap \Gamma)+e$ is not an independent set of $\mathbf{Q}$. Thus, since $M \cap \Gamma$ is an independent set of $\mathbf{Q}$, this and the definition of $\mathbf{Q}$ imply that $I+e$ is not an independent set of $\mathbf{M}_{r}$. Thus, Lemma 4 implies that $N(r)-h \in \mathcal{I}_{r}$.

\subsubsection{Completing a proof}

We are now ready to complete a proof of Lemma 10. If $N_{0}(r) \in \mathcal{I}_{r}$, then it follows from Lemmas 14, 15(i), and 16(ii) that $N_{0}$ is a matching in $G$. In addition, Lemma 15(ii) and 16(i) imply that $\phi\left(N_{0} ; M\right) \geq 1$, which contradicts the fact that $M$ is a popular matching in $G$. Thus, in the sequel, we can assume that $N_{0}(r) \notin \mathcal{I}_{r}$. In this case, since $N_{0}(r) \subseteq N(r)$, $N(r)$ is not in $\mathcal{I}_{r}$. Thus, Lemma 17 implies that $\mathbf{C}_{\mathbf{M}_{r}}(h, N(r)-h)$ and $\mathbf{C}_{\mathbf{M}_{r}}\left(h, N_{0}(r)-h\right)$ are well-defined. Define

$$
\begin{aligned}
C & :=\mathrm{C}_{\mathbf{M}_{r}}(h, N(r)-h), \\
C_{0} & :=\mathrm{C}_{\mathbf{M}_{r}}\left(h, N_{0}(r)-h\right) .
\end{aligned}
$$


Notice that Lemma 3 implies that $C=C_{0}$.

Since $N_{0} \cap \Gamma$ is an independent set of $\mathbf{Q}$, it follows from the definition of $\mathbf{Q}$ that $N_{0}(r) \cap \Gamma$ is in $\mathcal{I}_{r}$. Thus, $C_{0} \subseteq N_{0}(r)$ implies that $C_{0} \nsubseteq \Gamma$. Let $g_{1}=\left(b_{1}, r\right)$ be an edge in $C_{0} \backslash \Gamma$. Since $g \in \Gamma$ and $g_{1} \notin \Gamma$, we have $g \neq g_{1}$. Thus, since $g, g_{1} \in N_{0}$, we have $b \neq b_{1}$. Define $N_{1}:=N_{0}-g_{1}$. Then, $g_{1} \in C_{0}$ and Lemmas 14, 15(i), and 16(ii) imply that

- $\left|N_{1}(a)\right|=1$ for every applicant $a$ in $A-b_{1}$, and $N_{1}\left(b_{1}\right)=\emptyset$,

- $N_{1}(p) \in \mathcal{I}_{p}$ for every post $p$ in $P$.

Let $h_{1}=\left(b_{1}, q_{1}\right)$ be an edge in $f\left(b_{1}\right)$. We first consider the case where $N_{1}\left(q_{1}\right)+h_{1} \in \mathcal{I}_{q_{1}}$. Define $N_{2}:=N_{1}+h_{1}$. In this case, $N_{2}$ is a matching in $G$. Furthermore, since it follows from $g_{1} \notin \Gamma$, Lemmas 15 (ii) and 16(i) that

$$
\begin{aligned}
& \mu_{N_{2}}(b)=g \succ_{b} \mu_{M}(b), \\
& \mu_{N_{2}}\left(b_{1}\right)=h_{1} \succ_{b_{1}} g_{1}=\mu_{N}\left(b_{1}\right) \succsim_{b_{1}} \mu_{M}\left(b_{1}\right), \\
& \forall a \in A \backslash\left\{b, b_{1}\right\}: \mu_{N_{2}}(a)=\mu_{N}(a) \succsim_{a} \mu_{M}(a),
\end{aligned}
$$

we have $\phi\left(N_{2} ; M\right) \geq 2$. These contradict the fact that $M$ is a popular matching in $G$.

Next we consider the case where $N_{1}\left(q_{1}\right)+h_{1} \notin \mathcal{I}_{q_{1}}$. Define

$$
C_{1}:=\mathrm{C}_{\mathbf{M}_{q_{1}}}\left(h_{1}, N_{1}\left(q_{1}\right)\right) \text {. }
$$

We first assume that at least one of $q_{1} \neq q$ and $C_{1} \backslash\left\{g, h_{1}\right\} \neq \emptyset$ holds. Let $g_{2}=\left(b_{2}, q_{1}\right)$ be an edge in $C_{1} \backslash\left\{g, h_{1}\right\}$. Since $g_{2} \neq g, h_{1}$ and $g, g_{2} \in N_{1}$, we have $b_{2} \neq b, b_{1}$. Define

$$
N_{3}:=N_{2}-g_{2}+\ell\left(b_{2}\right) .
$$

Then, $N_{3}$ is a matching in $G$. Since Lemmas 15(ii) and 16(i) imply that

$$
\begin{aligned}
& \mu_{N_{3}}(b)=g \succ_{b} \mu_{M}(b), \\
& \mu_{N_{3}}\left(b_{1}\right)=h_{1} \succ_{b_{1}} g_{1}=\mu_{N}\left(b_{1}\right) \succsim_{b_{1}} \mu_{M}\left(b_{1}\right), \\
& \mu_{M}\left(b_{2}\right) \succ_{b_{2}} \ell\left(b_{2}\right)=\mu_{N_{3}}\left(b_{2}\right), \\
& \forall a \in A \backslash\left\{b, b_{1}, b_{2}\right\}: \mu_{N_{3}}(a)=\mu_{N}(a) \succsim_{a} \mu_{M}(a),
\end{aligned}
$$

we have $\phi\left(N_{3} ; M\right) \geq 1$. This contradicts the fact that $M$ is a popular matching in $G$.

Next we consider the case where $q_{1}=q$ and $C_{1}=\left\{g, h_{1}\right\}$. Define

$$
N_{4}:=N_{2}-g+\mu_{M}(b) .
$$

Assume that $\mu_{M}(b)=\left(b, q^{\prime}\right)$. Notice that $q^{\prime} \neq q$. If $q^{\prime}=r$, then

$$
N_{4}(r)=N_{2}(r)+\mu_{M}(b)=N_{1}(r)+\mu_{M}(b)=N_{0}(r)+\mu_{M}(b)-g_{1}=N(r)-g_{1} .
$$

Since $g_{1} \in C_{0}=C, N(r)-g_{1} \in \mathcal{I}_{r}$ and $N_{4}(r)$ is an independent set of $\mathbf{M}_{r}$. If $q^{\prime} \neq r$, then

$$
N_{4}\left(q^{\prime}\right)=N_{2}\left(q^{\prime}\right)+\mu_{M}(b)=N_{1}\left(q^{\prime}\right)+\mu_{M}(b)=N_{0}\left(q^{\prime}\right)+\mu_{M}(b)=N\left(q^{\prime}\right) .
$$


Lemma 14 implies that $N_{4}\left(q^{\prime}\right)$ is an independent set of $\mathbf{M}_{r}$. In both cases, $N_{4}$ is a matching in $G$. Furthermore, since Lemma 15(ii) implies that

$$
\begin{aligned}
& \mu_{N_{4}}(b)=\mu_{M}(b), \\
& \mu_{N_{4}}\left(b_{1}\right)=h_{1} \succ_{b_{1}} g_{1}=\mu_{N}\left(b_{1}\right) \succsim_{b_{1}} \mu_{M}\left(b_{1}\right), \\
& \forall a \in A \backslash\left\{b, b_{1}\right\}: \mu_{N_{4}}(a)=\mu_{N}(a) \succsim_{a} \mu_{M}(a),
\end{aligned}
$$

we have $\phi\left(N_{4} ; M\right) \geq 1$. This contradicts the fact that $M$ is a popular matching in $G$, and completes the proof.

\subsection{Proof of Lemma 12}

In this subsection, we give a proof of Lemma 12.

For proving this lemma by contradiction, we assume that there exists an applicant $b$ in $A$ such that $\mu_{M}(b) \notin \Pi$. Then, Lemma 11 implies that $e \succ_{b} \mu_{M}(b)$ for an edge $e$ in $s(b)$. Let $g=(b, q)$ be an edge in $s(b)$. Define $N:=M+g-\mu_{M}(b)$. Since $N(q)=M(q)+g$, if $M(q)+g \in \mathcal{I}_{q}$, then (I1) implies that $N$ is a matching in $G$. Furthermore, $\phi(N ; M)=1$. This contradicts the fact that $M$ is a popular matching in $G$. Thus, in the sequel, we can assume that $M(q)+g \notin \mathcal{I}_{q}$.

We first consider the case where

$$
\mathrm{C}_{\mathbf{M}_{q}}(g, M(q))-g \nsubseteq \Gamma \text {. }
$$

Let $e_{1}=\left(a_{1}, q\right)$ be an edge in

$$
\left(\mathrm{C}_{\mathbf{M}_{q}}(g, M(q))-g\right) \backslash \Gamma .
$$

Since $g \neq e_{1}$ holds, we have $a_{1} \neq b$. Let $e_{2}=\left(a_{1}, q_{1}\right)$ be an edge in $f\left(a_{1}\right)$. Since $e_{1} \notin \Gamma$ and $e_{2} \in \Gamma$ (i.e., $e_{1} \neq e_{2}$ ), we have $q_{1} \neq q$. Define $N^{\prime}:=N+e_{2}-e_{1}$. If $N\left(q_{1}\right)+e_{2} \in \mathcal{I}_{q_{1}}$, then $N^{\prime}$ is a matching in $G$. Furthermore, we have $\phi\left(N^{\prime} ; M\right)=2$, which contradicts the fact that $M$ is a popular matching in $G$. Assume that $N\left(q_{1}\right)+e_{2} \notin \mathcal{I}_{q_{1}}$. Let $e_{3}=\left(a_{2}, q_{1}\right)$ be an edge in $\mathrm{C}_{\mathbf{M}_{q_{1}}}\left(e_{2}, N\left(q_{1}\right)\right)-e_{2}$. Since $q_{1} \neq q$, we have $e_{3} \neq g$. Define $N^{\prime \prime}:=N^{\prime}-e_{3}+\ell\left(a_{2}\right)$. Then, $N^{\prime \prime}$ is a matching in $G$ and we have $\phi\left(N^{\prime \prime} ; M\right)=1$. This contradicts the fact that $M$ is a popular matching in $G$.

Next we consider the case where

$$
\mathrm{C}_{\mathbf{M}_{q}}(g, M(q))-g \subseteq \Gamma .
$$

Since $g$ is in $s(a)$, Lemmas 1,7 , and 10 imply

$$
\left(M \cap\left(\Omega_{\mathbf{B Q}}^{+} \cup \Omega_{\mathbf{B Q}}\right)\right)+g
$$

is an independent set of $\mathbf{P}$. This and the definition of $\mathbf{P}$ imply that

$$
\left(M(q) \cap\left(\Omega_{\mathbf{B Q}}^{+} \cup \Omega_{\mathbf{B Q}}\right)\right)+g \in \mathcal{I}_{q} .
$$

Thus,

$$
\mathrm{C}_{\mathbf{M}_{q}}(g, M(q))-g \not \subset\left(M(q) \cap\left(\Omega_{\mathbf{B Q}}^{+} \cup \Omega_{\mathbf{B Q}}\right)\right) .
$$


This implies that there exists an edge in $\mathbf{C}_{\mathbf{M}_{q}}(g, M(q)) \cap \Omega_{\mathbf{B Q}}^{-}$.

Since (8) implies that

$$
\mathrm{C}_{\mathbf{M}_{q}}(g, M(q)) \subseteq(M(q) \cap \Gamma)+g,
$$

we have $(M(q) \cap \Gamma)+g \notin \mathcal{I}_{q}$. Since (I1) and $M(q) \in \mathcal{I}_{q}$ imply that $M(q) \cap \Gamma \in \mathcal{I}_{q}$, this and Lemma 3 imply that

$$
\mathrm{C}_{\mathbf{M}_{q}}(g, M(q) \cap \Gamma)=\mathrm{C}_{\mathbf{M}_{q}}(g, M(q)) .
$$

Thus,

$$
\mathrm{C}_{\mathbf{M}_{q}}(g, M(q) \cap \Gamma) \cap \Omega_{\mathbf{B Q}}^{-} \neq \emptyset .
$$

Define $\mathbf{C}:=\mathbf{A} \mid(\Gamma+g)$ and $\mathbf{R}:=\mathbf{P} \mid(\Gamma+g)$. It is not difficult to see that $\mathbf{D}_{\mathbf{C R}}(M \cap \Gamma)$ is obtained from $\mathbf{D}_{\mathbf{B Q}}(M \cap \Gamma)$ as follows.

Step 1. Add $g$ to the vertex set of $\mathbf{D}_{\mathbf{B Q}}(M \cap \Gamma)$.

Step 2. Add an arc from $g$ to every edge in $\mathrm{C}_{\mathbf{M}_{q}}(g, M(q) \cap \Gamma)$.

Furthermore, since $\mu_{M}(b) \notin f(b)$, we have

$$
\begin{aligned}
& \mathrm{T}_{\mathbf{C}}(M \cap \Gamma)=\mathrm{T}_{\mathbf{B}}(M \cap \Gamma)+g, \\
& \mathrm{~T}_{\mathbf{R}}(M \cap \Gamma)=\mathrm{T}_{\mathbf{Q}}(M \cap \Gamma) .
\end{aligned}
$$

It follows from (9) that there exists an edge $h$ in $\mathrm{T}_{\mathbf{R}}(M \cap \Gamma)$ such that there a directed path in $\mathrm{D}_{\mathbf{C R}}(M \cap \Gamma)$ from $g$ to $h$. Let $L$ be a shortest directed path in $\mathrm{D}_{\mathbf{C R}}(M \cap \Gamma)$ from $g$ to $h$. We denote by $K=\left\{e_{1}, e_{2}, \ldots, e_{k}\right\}$ the set of edges in $\Gamma+g$ that $L$ goes through. Assume that $e_{i}:=\left(a_{i}, p_{i}\right)$ for each $i=1,2, \ldots, k$, and $L$ goes through $e_{1}, e_{2}, \ldots, e_{k}$ in this order. Notice that $e_{1}=g, e_{k}=h, k$ is odd, and $e_{i} \in M$ for every $i=2,4, \ldots, k-1$. Define $N:=M \triangle K$ and $N_{0}:=N-\mu_{M}(b)$. Lemma 6 implies that $N \cap(\Gamma+g)\left(=N_{0} \cap(\Gamma+g)\right)$ is an independent set of $\mathbf{R}$.

For completing a proof of Lemma 12, we first necessary lemmas (Section 3.2.1), and complete a proof of Lemma 12 (Section 3.2.2).

\subsubsection{Necessary lemmas}

Here we give necessary lemmas for completing the proof of Lemma 12.

Lemma 18. For every post $p$ in $P-r$, we have $N(p) \in \mathcal{I}_{p}$ and $N_{0}(p) \in \mathcal{I}_{p}$.

Proof. Let $p$ be a post in $P-r$. By using Lemma 4, we prove that $N(p) \in \mathcal{I}_{p}$. If we can prove this, then (I1) implies that $N_{0}(p) \in \mathcal{I}_{p}$.

Define $I:=M(p) \cap \Gamma, J:=M(p) \backslash \Gamma, X:=K(p) \backslash M$, and $Y:=M(p) \cap K$. Then,

$$
\begin{aligned}
(I \cup X) \backslash Y & =N(p) \cap(\Gamma+g), \\
((I \cup X) \backslash Y) \cup J & =N(p) .
\end{aligned}
$$

Since $M$ is an independent set of $\mathbf{P}$, the definition of $\mathbf{P}$ implies that $I \cup J$ is in $\mathcal{I}_{p}$. In addition, the definition of $\mathbf{R}$ implies that implies that $(I \cup X) \backslash Y$ is in $\mathcal{I}_{p}$. For every edge $e$ in $X$, the definition of $\mathbf{D}_{\mathbf{C R}}(M \cap \Gamma)$ implies that $(M \cap \Gamma)+e$ is not an independent set of $\mathbf{R}$. Thus, since $M \cap \Gamma$ is an independent set of $\mathbf{R}$, this and the definition of $\mathbf{R}$ imply that $I+e$ is not an independent set of $\mathbf{M}_{p}$. Thus, Lemma 4 implies that $N(p) \in \mathcal{I}_{p}$. 
Lemma 19. For every applicant $a$ in $A-b$, (i) $|N(a)|=1$, and (ii) $\mu_{N}(a) \succsim_{a} \mu_{M}(a)$.

Proof. For every $i=2,4, \ldots, k-1$, since there exists an arc in $\mathbf{D}_{\mathbf{C R}}(M \cap \Gamma)$ from $e_{i}$ to $e_{i+1}, M+e_{i+1}-e_{i}$ is an independent set of $\mathbf{C}$. Thus, the definitions of $\mathbf{A}$ implies that $a_{i}=a_{i+1}$ for every $i=2,4, \ldots, k-1$. This implies that for every applicant $a$ in $A-b$, since $|M(a)|=1$, we have $|N(a)|=1$. Furthermore, since $K-g \subseteq \Gamma, \mu_{N}(a) \in f(a)$ for every applicant $a$ in $A-b$, which implies (ii). This completes the proof.

Lemma 20. (i) $g \succ_{b} \mu_{M}(b)$, and (ii) $\left|N_{0}(b)\right|=1$.

Proof. Since $g \in s(b)$ and $e \succ_{b} \mu_{M}(b)$ for an edge $e$ in $s(b)$, we have $g \succ_{b} \mu_{M}(b)$. Furthermore, as proved in Lemma 19, we have $a_{i}=a_{i+1}$ for every $i=1,3, \ldots, k-1$. Thus, since $\mu_{M}(b) \notin \Gamma$, we have $K(b)=\{g\}$. This and $M(b)=\left\{\mu_{M}(b)\right\}$ imply (ii).

We denote by $L^{\prime}$ the subpath of $L$ from $g$ to $e_{k-1}$. Since $L$ is a shortest directed path in $\mathbf{D}_{\mathbf{C R}}(M \cap \Gamma)$ from $g$ to $h, L^{\prime}$ is a shortest directed path in $\mathbf{D}_{\mathbf{C R}}(M \cap \Gamma)$ from $g$ to $e_{k-1}$. Define $K^{\prime}:=K-h$. Then, $N-h=M \triangle K^{\prime}$. Lemma 6 implies that $(N-h) \cap(\Gamma+g)$ is an independent set of $\mathbf{R}$.

Lemma 21. $N(r)-h \in \mathcal{I}_{r}$ and $N_{0}(r)-h \in \mathcal{I}_{r}$.

Proof. By using Lemma 4, we prove that $N(r)-h \in \mathcal{I}_{r}$. If we can prove this, then (I1) implies that $N_{0}(r)-h \in \mathcal{I}_{r}$.

Define $I:=M(r) \cap \Gamma, J:=M(r) \backslash \Gamma, X:=K^{\prime}(r) \backslash M$, and $Y:=M(r) \cap K^{\prime}$. Then,

$$
\begin{aligned}
(I \cup X) \backslash Y & =(N(r)-h) \cap(\Gamma+g), \\
((I \cup X) \backslash Y) \cup J & =N(r)-h .
\end{aligned}
$$

Since $M$ is an independent set of $\mathbf{P}$, the definition of $\mathbf{P}$ implies that $I \cup J$ is in $\mathcal{I}_{r}$. In addition, the definition of $\mathbf{R}$ implies that $(I \cup X) \backslash Y$ is in $\mathcal{I}_{r}$. For every edge $e$ in $X$, the definition of $\mathbf{D}_{\mathbf{C R}}(M \cap \Gamma)$ implies that $(M \cap \Gamma)+e$ is not an independent set of $\mathbf{R}$. Thus, since $M \cap \Gamma$ is an independent set of $\mathbf{R}$, this and the definition of $\mathbf{R}$ imply that $I+e$ is not an independent set of $\mathbf{M}_{r}$. Thus, Lemma 4 implies that $N(r)-h \in \mathcal{I}_{r}$.

\subsubsection{Completing a proof}

We are now ready to complete a proof of Lemma 12. If $N_{0}(r) \in \mathcal{I}_{r}$, then it follows from Lemmas 18, 19(i), and 20(ii) that $N_{0}$ is a matching in $G$. Furthermore, Lemma 19(ii) and 20(i) imply that $\phi\left(N_{0} ; M\right) \geq 1$, which contradicts the fact that $M$ is a popular matching in $G$. Thus, in the sequel, we can assume that $N_{0}(r) \notin \mathcal{I}_{r}$. In this case, since $N_{0}(r) \subseteq N(r)$, $N(r)$ is not in $\mathcal{I}_{r}$. Lemma 21 implies that $\mathbf{C}_{\mathbf{M}_{r}}(h, N(r)-h)$ and $\mathbf{C}_{\mathbf{M}_{r}}\left(h, N_{0}(r)-h\right)$ are well-defined. Define

$$
\begin{aligned}
C & :=\mathrm{C}_{\mathbf{M}_{r}}(h, N(r)-h), \\
C_{0} & :=\mathrm{C}_{\mathbf{M}_{r}}\left(h, N_{0}(r)-h\right) .
\end{aligned}
$$

Lemma 3 implies that $C=C_{0}$.

Since $N_{0} \cap(\Gamma+g)$ is an independent set of $\mathbf{R}$, the definition of $\mathbf{R}$ implies that $N_{0}(r) \cap$ $(\Gamma+g)$ is in $\mathcal{I}_{r}$. Thus, if $C_{0} \subseteq \Gamma+g$, then $C_{0} \subseteq N_{0}(r)$ implies that $C_{0} \subseteq N_{0}(r) \cap(\Gamma+g)$. 
This contradicts the fact that $N_{0}(r) \cap(\Gamma+g) \in \mathcal{I}_{r}$. Thus, $C_{0} \not \subset \Gamma+g$. Let $g_{1}=\left(b_{1}, r\right)$ be an edge in $C_{0} \backslash(\Gamma+g)$. Notice that since $g \neq g_{1}$ and $g, g_{1} \in N_{0}$, we have $b \neq b_{1}$. Define $N_{1}:=N_{0}-g_{1}$. Then, $g_{1} \in C_{0}$ and Lemmas 18, 19(i), and 20(ii) imply that

- $\left|N_{1}(a)\right|=1$ for every applicant $a$ in $A-b_{1}$, and $N_{1}\left(b_{1}\right)=\emptyset$,

- $N_{1}(p) \in \mathcal{I}_{p}$ for every post $p$ in $P$.

Let $h_{1}=\left(b_{1}, q_{1}\right)$ be an edge in $f\left(b_{1}\right)$. We first consider the case where $N_{1}\left(q_{1}\right)+h_{1} \in \mathcal{I}_{q_{1}}$. Define $N_{2}:=N_{1}+h_{1}$. In this case, $N_{2}$ is a matching in $G$. Furthermore, since it follows from $g_{1} \notin \Gamma$, Lemmas 19 (ii) and 20(i) that

$$
\begin{aligned}
& \mu_{N_{2}}(b)=g \succ_{b} \mu_{M}(b), \\
& \mu_{N_{2}}\left(b_{1}\right)=h_{1} \succ_{b_{1}} g_{1}=\mu_{N}\left(b_{1}\right) \succsim_{b_{1}} \mu_{M}\left(b_{1}\right), \\
& \forall a \in A \backslash\left\{b, b_{1}\right\}: \mu_{N_{2}}(a)=\mu_{N}(a) \succsim_{a} \mu_{M}(a),
\end{aligned}
$$

we have $\phi\left(N_{2} ; M\right) \geq 2$. These contradict the fact that $M$ is a popular matching in $G$.

Next we consider the case where $N_{1}\left(q_{1}\right)+h_{1} \notin \mathcal{I}_{q_{1}}$. Define

$$
C_{1}:=\mathrm{C}_{\mathbf{M}_{q_{1}}}\left(h_{1}, N_{1}\left(q_{1}\right)\right) \text {. }
$$

We first assume that at least one of $q_{1} \neq q$ and $C_{1} \backslash\left\{g, h_{1}\right\} \neq \emptyset$ holds. Let $g_{2}=\left(b_{2}, q_{1}\right)$ be an edge in $C_{1} \backslash\left\{g, h_{1}\right\}$. Since $g_{2} \neq g, h_{1}$ and $g, g_{2} \in N_{1}$, we have $b_{2} \neq b, b_{1}$. Define

$$
N_{3}:=N_{2}-g_{2}+\ell\left(b_{2}\right) .
$$

Then, $N_{3}$ is a matching in $G$. Since Lemmas 19(ii) and 20(i) imply that

$$
\begin{aligned}
& \mu_{N_{3}}(b)=g \succ_{b} \mu_{M}(b), \\
& \mu_{N_{3}}\left(b_{1}\right)=h_{1} \succ_{b_{1}} g_{1}=\mu_{N}\left(b_{1}\right) \succsim_{b_{1}} \mu_{M}\left(b_{1}\right), \\
& \mu_{M}\left(b_{2}\right) \succ_{b_{2}} \ell\left(b_{2}\right)=\mu_{N_{3}}\left(b_{2}\right), \\
& \forall a \in A \backslash\left\{b, b_{1}, b_{2}\right\}: \mu_{N_{3}}(a)=\mu_{N}(a) \succsim_{a} \mu_{M}(a),
\end{aligned}
$$

we have $\phi\left(N_{3} ; M\right) \geq 1$. This contradicts the fact that $M$ is a popular matching in $G$.

Next we consider the case where $q_{1}=q$ and $C_{1}=\left\{g, h_{1}\right\}$. Define

$$
N_{4}:=N_{2}-g+\mu_{M}(b) .
$$

Assume that $\mu_{M}(b)=\left(b, q^{\prime}\right)$. Notice that $q^{\prime} \neq q$. If $q^{\prime}=r$, then

$$
N_{4}(r)=N_{2}(r)+\mu_{M}(b)=N_{1}(r)+\mu_{M}(b)=N_{0}(r)+\mu_{M}(b)-g_{1}=N(r)-g_{1} .
$$

Since $g_{1} \in C_{0}=C, N(r)-g_{1} \in \mathcal{I}_{r}$ and $N_{4}(r)$ is an independent set of $\mathbf{M}_{r}$. If $q^{\prime} \neq r$, then

$$
N_{4}\left(q^{\prime}\right)=N_{2}\left(q^{\prime}\right)+\mu_{M}(b)=N_{1}\left(q^{\prime}\right)+\mu_{M}(b)=N_{0}\left(q^{\prime}\right)+\mu_{M}(b)=N\left(q^{\prime}\right) .
$$

Lemma 18 implies that $N_{4}\left(q^{\prime}\right)$ is an independent set of $\mathbf{M}_{r}$. In both cases, $N_{4}$ is a matching in $G$. Furthermore, since Lemma 19(ii) implies that

$$
\begin{aligned}
& \mu_{N_{4}}(b)=\mu_{M}(b), \\
& \mu_{N_{4}}\left(b_{1}\right)=h_{1} \succ_{b_{1}} g_{1}=\mu_{N}\left(b_{1}\right) \succsim_{b_{1}} \mu_{M}\left(b_{1}\right), \\
& \forall a \in A \backslash\left\{b, b_{1}\right\}: \mu_{N_{4}}(a)=\mu_{N}(a) \succsim_{a} \mu_{M}(a),
\end{aligned}
$$

we have $\phi\left(N_{4} ; M\right) \geq 1$, which contradicts the fact that $M$ is a popular matching in $G$. This completes the proof. 


\section{Algorithm}

In this section, we propose our algorithm for PMTM. Define a function $\xi: \Pi \rightarrow\{0,1\}$ by

$$
\xi(e):= \begin{cases}1 & \text { if } e \in \Gamma \\ 0 & \text { if } e \in \Pi \backslash \Gamma .\end{cases}
$$

Our algorithm PMTM is described as follows.

\section{$\underline{\text { Algorithm PMTM }}$}

Step 1. Compute $\Pi$, and define $\mathbf{A}^{\prime}:=\mathbf{A} \mid \Pi$ and $\mathbf{P}^{\prime}:=\mathbf{P} \mid \Pi$.

Step 2. Find a maximum-size common independent set $M$ of $\mathbf{A}^{\prime}$ and $\mathbf{P}^{\prime}$ such that

$$
\xi(M)=\max \left\{\xi(N) \mid N \in \mathcal{B}_{\mathbf{A}^{\prime} \mathbf{P}^{\prime}}\right\}
$$

Step 3. If $\xi(M)=\gamma_{\mathbf{B Q}}$ and $|M|=|A|$ hold, then output $M$ and halt (in this case, $M$ is a popular matching in $G$ ). Otherwise, output null and halt (in this case, there exists no popular matching in $G$ ).

\section{End of Algorithm}

For proving the correctness of the algorithm PMTM, we need the following lemma.

Lemma 22. Assume that we are given a popular matching $M$ in $G$. Then, $M$ is a common independent set of $\mathbf{A}^{\prime}$ and $\mathbf{P}^{\prime}$ such that $\xi(M)=\gamma_{\mathbf{B Q}}$ and $|M|=|A|$.

Proof. Since $M$ is a matching in $G, M$ is a common independent set of $\mathbf{A}$ and $\mathbf{P}$ such that $|M|=|A|$. In addition, (P2) of Theorem 9 implies that $M$ is a subset of $\Pi$. Thus, $M$ is a common independent set of $\mathbf{A}^{\prime}$ and $\mathbf{P}^{\prime}$. Since (P1) of Theorem 9 implies that $M \cap \Gamma$ is a maximum-size common independent set of $\mathbf{B}$ and $\mathbf{Q}$, we have $|M \cap \Gamma|=\gamma_{\mathbf{B} \mathbf{Q}}$, i.e., $\xi(M)=\gamma_{\mathbf{B Q}}$. This completes the proof.

We are now ready to prove the correctness of the algorithm PMTM.

Theorem 23. The algorithm PMTM can correctly solve PMTM.

Proof. Let $M$ be a common independent set of $\mathbf{A}^{\prime}$ and $\mathbf{P}^{\prime}$ that is found in Step $\mathbf{2}$ of the algorithm PMTM. If the algorithm PMTM outputs $M$, then $M$ is a matching and Theorem 9 implies that $M$ is a popular matching in $G$.

Assume that the algorithm PMTM outputs null. Since $M$ is a common independent set of $\mathbf{A}$ and $\mathbf{P}$, (I1) implies that $M \cap \Gamma$ is a common independent set of $\mathbf{B}$ and $\mathbf{Q}$. Thus, $|M \cap \Gamma| \leq \gamma_{\mathbf{B Q}}$, which implies that $\xi(M) \leq \gamma_{\mathbf{B Q}}$. Furthermore, since $|M(a)| \leq 1$ for every applicant $a$ in $A$, we have $|M| \leq|A|$. Thus, $\xi(M)<\gamma_{\mathbf{B Q}}$ and/or $|M|<|A|$. We prove that in this case, there exists no popular matching in $G$ by contradiction. Assume that there exists a popular matching $N$ in $G$. Then, Lemma 22 implies that $N$ is a common independent set of $\mathbf{A}^{\prime}$ and $\mathbf{P}^{\prime}$ such that $\xi(N)=\gamma_{\mathbf{B Q}}$ and $|N|=|A|$. If $|M|<|A|$, then the existence of $N$ contradicts the fact that $M$ is a maximum-size common independent 
set of $\mathbf{A}^{\prime}$ and $\mathbf{P}^{\prime}$. If $\xi(M)<\gamma_{\mathbf{B Q}}$ and $|M|=|A|$, then the existence of $N$ contradicts the fact that

$$
\xi(M)=\max \left\{\xi\left(N^{\prime}\right) \mid N^{\prime} \in \mathcal{B}_{\mathbf{A}^{\prime} \mathbf{P}^{\prime}}\right\}
$$

Thus, there exists no popular matching in $G$. This completes the proof.

Here we consider the time complexity of the algorithm PMTM. We denote by EO the time required to decide whether $I+e \in \mathcal{I}_{p}$ for every post $p$ in $P$, every independent set $I$ of $\mathbf{M}_{p}$, and every edge $e$ in $E(p) \backslash I$. Define $m:=|E|$. For simplicity, we assume that $E(p) \neq \emptyset$ for every post $p$ in $P$ and $\mathrm{EO}=\Omega(m)$. Furthermore, we assume that for every applicant $a$ in $A$ and every edges $e, g$ in $E(a)$, we can decide in $O(1)$ time whether $e \succsim_{a} g$ holds.

We first consider the time complexity of Step 1. It is not difficult to see that we can compute $f(a)$ for all applicants $a$ in $O(m)$ time. For computing $s(a)$ for all applicants $a$ in $A$, we first compute $\Omega_{\mathbf{B Q}}^{+}, \Omega_{\mathbf{B Q}}$, and $\Omega_{\mathbf{B Q}}^{-}$in $O\left(m^{2.5} \mathrm{EO}\right)$ time by finding a maximum-size common independent set of $\mathbf{B}$ and $\mathbf{Q}$ via the algorithm of Cunningham [2]. Then, we find a base $B$ of $\mathbf{P} \mid\left(\Omega_{\mathbf{B Q}}^{+} \cup \Omega_{\mathbf{B Q}}\right)$ in $O(m \mathrm{EO})$ time. Lemma 1 implies that by using the base $B$, we can compute $s(a)$ for all applicants $a$ in $A$ in $O(m \mathrm{EO})$ time. Thus, we can compute $\Pi$ in $O\left(m^{2.5} \mathrm{EO}\right)$ time.

Next we consider the time complexity of Step 2. It is not difficult to see that we can decide in $O(m)$ time whether $M+e$ is an independent set of $\mathbf{A}^{\prime}$ for every independent set $M$ of $\mathbf{A}^{\prime}$ and every edge $e$ in $\Pi \backslash M$. Furthermore, it is not difficult to see that we can decide in $O(\mathrm{EO})$ time whether $M+e$ is an independent set of $\mathbf{P}^{\prime}$ for every independent set $M$ of $\mathbf{P}^{\prime}$ and every edge $e$ in $\Pi \backslash M$. Thus, in Step $\mathbf{2}$, we can find a desired maximumsize common independent set of $\mathbf{A}^{\prime}$ and $\mathbf{P}^{\prime}$ in $O\left(\mathrm{~m}^{3} \mathrm{EO}\right)$ time by using the algorithm of Frank [7]. Thus, the time complexity of the algorithm PMTM is $O\left(m^{3} \mathrm{EO}\right)$.

\section{References}

[1] D. J. Abraham, R. W. Irving, T. Kavitha, and K. Mehlhorn. Popular matchings. SIAM Journal on Computing, 37(4):1030-1045, 2007.

[2] W. H. Cunningham. Improved bounds for matroid partition and intersection algorithms. SIAM Journal on Computing, 15(4):948-957, 1986.

[3] A. L. Dulmage and N. S. Mendelsohn. Coverings of bipartite graphs. Canadian Journal of Mathematics, 10:517-534, 1958.

[4] A. L. Dulmage and N. S. Mendelsohn. A structure theory of bipartite graphs of finite exterior dimension. Transactions of the Royal Society of Canada, Section III, 53:1-13, 1959.

[5] J. Edmonds. Paths, trees, and flowers. Canadian Journal of Mathematics, 17:449467, 1965.

[6] T. Fleiner. A fixed-point approach to stable matchings and some applications. Mathematics of Operations Research, 28(1):103-126, 2003. 
[7] A. Frank. A weighted matroid intersection algorithm. Journal of Algorithms, 2(4):328-336, 1981.

[8] T. Gallai. Kritische graphen II. A Magyar Tudományos Akadémia-Matematikai Kutató Intézetének Közleményei, 8:373-395, 1963.

[9] T. Gallai. Maximale systeme unabhänginger kanten. A Magyar Tudományos Akadémia - Matematikai Kutató Intézetének Közleményei, 9:401-413, 1964.

[10] P. Gärdenfors. Match making: Assignments based on bilateral preferences. Behavioral Science, 20(3):166-173, 1975.

[11] N. Kamiyama. Matroid intersection with priority constraints. Journal of the Operations Research Society of Japan, 56(1):15-25, 2013.

[12] N. Kamiyama. The popular matching and condensation problems under matroid constraints. In Proceedings of the 8th Annual International Conference on Combinatorial Optimization and Applications, volume 8881 of Lecture Notes in Computer Science, pages 713-728, 2014.

[13] T. Kavitha, J. Mestre, and M. Nasre. Popular mixed matchings. Theoretical Computer Science, 412(24):2679-2690, 2011.

[14] T. Kavitha and M. Nasre. Optimal popular matchings. Discrete Applied Mathematics, 157(14):3181-3186, 2009.

[15] T. Kavitha and M. Nasre. Popular matchings with variable item copies. Theoretical Computer Science, 412(12):1263-1274, 2011.

[16] T. Kavitha, M. Nasre, and P. Nimbhorkar. Popularity at minimum cost. Journal of Combinatorial Optimization, 27(3):574-596, 2014.

[17] B. Korte and J. Vygen. Combinatorial Optimization: Theory and Algorithms. Springer, 5th edition, 2012.

[18] L. Lovász and M. D. Plummer. Matching Theory. North-Holland, 1986.

[19] D. F. Manlove and C. T. S. Sng. Popular matchings in the capacitated house allocation problem. In Proceedings of the 14th European Symposium on Algorithms, volume 4168 of Lecture Notes in Computer Science, pages 492-503, 2006.

[20] E. McDermid and R. W. Irving. Popular matchings: structure and algorithms. Journal of Combinatorial Optimization, 22(3):339-358, 2011.

[21] J. Mestre. Weighted popular matchings. ACM Transactions on Algorithms, 10(1):2:1-2:16, 2014.

[22] K. Murota. Matrices and Matroids for Systems Analysis. Springer, 2000.

[23] J. G. Oxley. Matroid theory. Oxford University Press, 2nd edition, 2011. 
[24] C. T. S. Sng and D. F. Manlove. Popular matchings in the weighted capacitated house allocation problem. Journal of Discrete Algorithms, 8(2):102-116, 2010.

[25] Y.-W. Wu, W.-Y. Lin, H.-L. Wang, and K.-M. Chao. An optimal algorithm for the popular condensation problem. In Proceedings of the 24th International Workshop on Combinatorial Algorithms, volume 8288 of Lecture Notes in Computer Science, pages 412-422, 2013.

[26] Y.-W. Wu, W.-Y. Lin, H.-L. Wang, and K.-M. Chao. The generalized popular condensation problem. In Proceedings of the 25th International Symposium on Algorithms and Computation, volume 8889 of Lecture Notes in Computer Science, pages 606-617, 2014.

[27] R. Zenklusen. Matroidal degree-bounded minimum spanning trees. In Proceedings of the 23rd Annual ACM-SIAM Symposium on Discrete Algorithms, pages 1512-1521, 2012. 


\section{List of MI Preprint Series, Kyushu University \\ The Global COE Program \\ Math-for-Industry Education \& Research Hub}

MI

MI2008-1 Takahiro ITO, Shuichi INOKUCHI \& Yoshihiro MIZOGUCHI

Abstract collision systems simulated by cellular automata

MI2008-2 Eiji ONODERA

The intial value problem for a third-order dispersive flow into compact almost Hermitian manifolds

MI2008-3 Hiroaki KIDO

On isosceles sets in the 4-dimensional Euclidean space

MI2008-4 Hirofumi NOTSU

Numerical computations of cavity flow problems by a pressure stabilized characteristiccurve finite element scheme

MI2008-5 Yoshiyasu OZEKI

Torsion points of abelian varieties with values in nfinite extensions over a p-adic field

MI2008-6 Yoshiyuki TOMIYAMA

Lifting Galois representations over arbitrary number fields

MI2008-7 Takehiro HIROTSU \& Setsuo TANIGUCHI

The random walk model revisited

MI2008-8 Silvia GANDY, Masaaki KANNO, Hirokazu ANAI \& Kazuhiro YOKOYAMA

Optimizing a particular real root of a polynomial by a special cylindrical algebraic decomposition

MI2008-9 Kazufumi KIMOTO, Sho MATSUMOTO \& Masato WAKAYAMA

Alpha-determinant cyclic modules and Jacobi polynomials

MI2008-10 Sangyeol LEE \& Hiroki MASUDA

Jarque-Bera Normality Test for the Driving Lévy Process of a Discretely Observed Univariate SDE

MI2008-11 Hiroyuki CHIHARA \& Eiji ONODERA

A third order dispersive flow for closed curves into almost Hermitian manifolds

MI2008-12 Takehiko KINOSHITA, Kouji HASHIMOTO and Mitsuhiro T. NAKAO

On the $L^{2}$ a priori error estimates to the finite element solution of elliptic problems with singular adjoint operator

MI2008-13 Jacques FARAUT and Masato WAKAYAMA

Hermitian symmetric spaces of tube type and multivariate Meixner-Pollaczek polynomials 
MI2008-14 Takashi NAKAMURA

Riemann zeta-values, Euler polynomials and the best constant of Sobolev inequality

MI2008-15 Takashi NAKAMURA

Some topics related to Hurwitz-Lerch zeta functions

MI2009-1 Yasuhide FUKUMOTO

Global time evolution of viscous vortex rings

MI2009-2 Hidetoshi MATSUI \& Sadanori KONISHI

Regularized functional regression modeling for functional response and predictors

MI2009-3 Hidetoshi MATSUI \& Sadanori KONISHI

Variable selection for functional regression model via the $L_{1}$ regularization

MI2009-4 Shuichi KAWANO \& Sadanori KONISHI

Nonlinear logistic discrimination via regularized Gaussian basis expansions

MI2009-5 Toshiro HIRANOUCHI \& Yuichiro TAGUCHII

Flat modules and Groebner bases over truncated discrete valuation rings

MI2009-6 Kenji KAJIWARA \& Yasuhiro OHTA

Bilinearization and Casorati determinant solutions to non-autonomous $1+1$ dimensional discrete soliton equations

MI2009-7 Yoshiyuki KAGEI

Asymptotic behavior of solutions of the compressible Navier-Stokes equation around the plane Couette flow

MI2009-8 Shohei TATEISHI, Hidetoshi MATSUI \& Sadanori KONISHI

Nonlinear regression modeling via the lasso-type regularization

MI2009-9 Takeshi TAKAISHI \& Masato KIMURA

Phase field model for mode III crack growth in two dimensional elasticity

MI2009-10 Shingo SAITO

Generalisation of Mack's formula for claims reserving with arbitrary exponents for the variance assumption

MI2009-11 Kenji KAJIWARA, Masanobu KANEKO, Atsushi NOBE \& Teruhisa TSUDA Ultradiscretization of a solvable two-dimensional chaotic map associated with the Hesse cubic curve

MI2009-12 Tetsu MASUDA

Hypergeometric $\tau$-functions of the q-Painlevé system of type $E_{8}^{(1)}$

MI2009-13 Hidenao IWANE, Hitoshi YANAMI, Hirokazu ANAI \& Kazuhiro YOKOYAMA A Practical Implementation of a Symbolic-Numeric Cylindrical Algebraic Decomposition for Quantifier Elimination

MI2009-14 Yasunori MAEKAWA

On Gaussian decay estimates of solutions to some linear elliptic equations and its applications 
MI2009-15 Yuya ISHIHARA \& Yoshiyuki KAGEI

Large time behavior of the semigroup on $L^{p}$ spaces associated with the linearized compressible Navier-Stokes equation in a cylindrical domain

MI2009-16 Chikashi ARITA, Atsuo KUNIBA, Kazumitsu SAKAI \& Tsuyoshi SAWABE

Spectrum in multi-species asymmetric simple exclusion process on a ring

MI2009-17 Masato WAKAYAMA \& Keitaro YAMAMOTO

Non-linear algebraic differential equations satisfied by certain family of elliptic functions

MI2009-18 Me Me NAING \& Yasuhide FUKUMOTO

Local Instability of an Elliptical Flow Subjected to a Coriolis Force

MI2009-19 Mitsunori KAYANO \& Sadanori KONISHI

Sparse functional principal component analysis via regularized basis expansions and its application

MI2009-20 Shuichi KAWANO \& Sadanori KONISHI

Semi-supervised logistic discrimination via regularized Gaussian basis expansions

MI2009-21 Hiroshi YOSHIDA, Yoshihiro MIWA \& Masanobu KANEKO

Elliptic curves and Fibonacci numbers arising from Lindenmayer system with symbolic computations

MI2009-22 Eiji ONODERA

A remark on the global existence of a third order dispersive flow into locally Hermitian symmetric spaces

MI2009-23 Stjepan LUGOMER \& Yasuhide FUKUMOTO

Generation of ribbons, helicoids and complex scherk surface in laser-matter Interactions

MI2009-24 Yu KAWAKAMI

Recent progress in value distribution of the hyperbolic Gauss map

MI2009-25 Takehiko KINOSHITA \& Mitsuhiro T. NAKAO

On very accurate enclosure of the optimal constant in the a priori error estimates for $H_{0}^{2}$-projection

MI2009-26 Manabu YOSHIDA

Ramification of local fields and Fontaine's property (Pm)

MI2009-27 Yu KAWAKAMI

Value distribution of the hyperbolic Gauss maps for flat fronts in hyperbolic threespace

MI2009-28 Masahisa TABATA

Numerical simulation of fluid movement in an hourglass by an energy-stable finite element scheme

MI2009-29 Yoshiyuki KAGEI \& Yasunori MAEKAWA

Asymptotic behaviors of solutions to evolution equations in the presence of translation and scaling invariance 
MI2009-30 Yoshiyuki KAGEI \& Yasunori MAEKAWA

On asymptotic behaviors of solutions to parabolic systems modelling chemotaxis

MI2009-31 Masato WAKAYAMA \& Yoshinori YAMASAKI

Hecke's zeros and higher depth determinants

MI2009-32 Olivier PIRONNEAU \& Masahisa TABATA

Stability and convergence of a Galerkin-characteristics finite element scheme of lumped mass type

MI2009-33 Chikashi ARITA

Queueing process with excluded-volume effect

MI2009-34 Kenji KAJIWARA, Nobutaka NAKAZONO \& Teruhisa TSUDA

Projective reduction of the discrete Painlevé system of type $\left(A_{2}+A_{1}\right)^{(1)}$

MI2009-35 Yosuke MIZUYAMA, Takamasa SHINDE, Masahisa TABATA \& Daisuke TAGAMI Finite element computation for scattering problems of micro-hologram using DtN map

MI2009-36 Reiichiro KAWAI \& Hiroki MASUDA

Exact simulation of finite variation tempered stable Ornstein-Uhlenbeck processes

MI2009-37 Hiroki MASUDA

On statistical aspects in calibrating a geometric skewed stable asset price model

MI2010-1 Hiroki MASUDA

Approximate self-weighted LAD estimation of discretely observed ergodic OrnsteinUhlenbeck processes

MI2010-2 Reiichiro KAWAI \& Hiroki MASUDA

Infinite variation tempered stable Ornstein-Uhlenbeck processes with discrete observations

MI2010-3 Kei HIROSE, Shuichi KAWANO, Daisuke MIIKE \& Sadanori KONISHI

Hyper-parameter selection in Bayesian structural equation models

MI2010-4 Nobuyuki IKEDA \& Setsuo TANIGUCHI

The Itô-Nisio theorem, quadratic Wiener functionals, and 1-solitons

MI2010-5 Shohei TATEISHI \& Sadanori KONISHI

Nonlinear regression modeling and detecting change point via the relevance vector machine

MI2010-6 Shuichi KAWANO, Toshihiro MISUMI \& Sadanori KONISHI

Semi-supervised logistic discrimination via graph-based regularization

MI2010-7 Teruhisa TSUDA

UC hierarchy and monodromy preserving deformation

MI2010-8 Takahiro ITO

Abstract collision systems on groups 
MI2010-9 Hiroshi YOSHIDA, Kinji KIMURA, Naoki YOSHIDA, Junko TANAKA \& Yoshihiro MIWA

An algebraic approach to underdetermined experiments

MI2010-10 Kei HIROSE \& Sadanori KONISHI

Variable selection via the grouped weighted lasso for factor analysis models

MI2010-11 Katsusuke NABESHIMA \& Hiroshi YOSHIDA

Derivation of specific conditions with Comprehensive Groebner Systems

MI2010-12 Yoshiyuki KAGEI, Yu NAGAFUCHI \& Takeshi SUDOU

Decay estimates on solutions of the linearized compressible Navier-Stokes equation around a Poiseuille type flow

MI2010-13 Reiichiro KAWAI \& Hiroki MASUDA

On simulation of tempered stable random variates

MI2010-14 Yoshiyasu OZEKI

Non-existence of certain Galois representations with a uniform tame inertia weight

MI2010-15 Me Me NAING \& Yasuhide FUKUMOTO

Local Instability of a Rotating Flow Driven by Precession of Arbitrary Frequency

MI2010-16 Yu KAWAKAMI \& Daisuke NAKAJO

The value distribution of the Gauss map of improper affine spheres

MI2010-17 Kazunori YASUTAKE

On the classification of rank 2 almost Fano bundles on projective space

MI2010-18 Toshimitsu TAKAESU

Scaling limits for the system of semi-relativistic particles coupled to a scalar bose field

MI2010-19 Reiichiro KAWAI \& Hiroki MASUDA

Local asymptotic normality for normal inverse Gaussian Lévy processes with highfrequency sampling

MI2010-20 Yasuhide FUKUMOTO, Makoto HIROTA \& Youichi MIE

Lagrangian approach to weakly nonlinear stability of an elliptical flow

MI2010-21 Hiroki MASUDA

Approximate quadratic estimating function for discretely observed Lévy driven SDEs with application to a noise normality test

MI2010-22 Toshimitsu TAKAESU

A Generalized Scaling Limit and its Application to the Semi-Relativistic Particles System Coupled to a Bose Field with Removing Ultraviolet Cutoffs

MI2010-23 Takahiro ITO, Mitsuhiko FUJIO, Shuichi INOKUCHI \& Yoshihiro MIZOGUCHI Composition, union and division of cellular automata on groups

MI2010-24 Toshimitsu TAKAESU

A Hardy's Uncertainty Principle Lemma in Weak Commutation Relations of HeisenbergLie Algebra 
MI2010-25 Toshimitsu TAKAESU

On the Essential Self-Adjointness of Anti-Commutative Operators

MI2010-26 Reiichiro KAWAI \& Hiroki MASUDA

On the local asymptotic behavior of the likelihood function for Meixner Lévy processes under high-frequency sampling

MI2010-27 Chikashi ARITA \& Daichi YANAGISAWA

Exclusive Queueing Process with Discrete Time

MI2010-28 Jun-ichi INOGUCHI, Kenji KAJIWARA, Nozomu MATSUURA \& Yasuhiro OHTA Motion and Bäcklund transformations of discrete plane curves

MI2010-29 Takanori YASUDA, Masaya YASUDA, Takeshi SHIMOYAMA \& Jun KOGURE On the Number of the Pairing-friendly Curves

MI2010-30 Chikashi ARITA \& Kohei MOTEGI

Spin-spin correlation functions of the $q$-VBS state of an integer spin model

MI2010-31 Shohei TATEISHI \& Sadanori KONISHI

Nonlinear regression modeling and spike detection via Gaussian basis expansions

MI2010-32 Nobutaka NAKAZONO

Hypergeometric $\tau$ functions of the $q$-Painlevé systems of type $\left(A_{2}+A_{1}\right)^{(1)}$

MI2010-33 Yoshiyuki KAGEI

Global existence of solutions to the compressible Navier-Stokes equation around parallel flows

MI2010-34 Nobushige KUROKAWA, Masato WAKAYAMA \& Yoshinori YAMASAKI

Milnor-Selberg zeta functions and zeta regularizations

MI2010-35 Kissani PERERA \& Yoshihiro MIZOGUCHI

Laplacian energy of directed graphs and minimizing maximum outdegree algorithms

MI2010-36 Takanori YASUDA

CAP representations of inner forms of $S p(4)$ with respect to Klingen parabolic subgroup

MI2010-37 Chikashi ARITA \& Andreas SCHADSCHNEIDER

Dynamical analysis of the exclusive queueing process

MI2011-1 Yasuhide FUKUMOTO\& Alexander B. SAMOKHIN

Singular electromagnetic modes in an anisotropic medium

MI2011-2 Hiroki KONDO, Shingo SAITO \& Setsuo TANIGUCHI

Asymptotic tail dependence of the normal copula

MI2011-3 Takehiro HIROTSU, Hiroki KONDO, Shingo SAITO, Takuya SATO, Tatsushi TANAKA \& Setsuo TANIGUCHI

Anderson-Darling test and the Malliavin calculus

MI2011-4 Hiroshi INOUE, Shohei TATEISHI \& Sadanori KONISHI

Nonlinear regression modeling via Compressed Sensing 
MI2011-5 Hiroshi INOUE

Implications in Compressed Sensing and the Restricted Isometry Property

MI2011-6 Daeju KIM \& Sadanori KONISHI

Predictive information criterion for nonlinear regression model based on basis expansion methods

MI2011-7 Shohei TATEISHI, Chiaki KINJYO \& Sadanori KONISHI

Group variable selection via relevance vector machine

MI2011-8 Jan BREZINA \& Yoshiyuki KAGEI

Decay properties of solutions to the linearized compressible Navier-Stokes equation around time-periodic parallel flow

Group variable selection via relevance vector machine

MI2011-9 Chikashi ARITA, Arvind AYYER, Kirone MALLICK \& Sylvain PROLHAC

Recursive structures in the multispecies TASEP

MI2011-10 Kazunori YASUTAKE

On projective space bundle with nef normalized tautological line bundle

MI2011-11 Hisashi ANDO, Mike HAY, Kenji KAJIWARA \& Tetsu MASUDA

An explicit formula for the discrete power function associated with circle patterns of Schramm type

MI2011-12 Yoshiyuki KAGEI

Asymptotic behavior of solutions to the compressible Navier-Stokes equation around a parallel flow

MI2011-13 Vladimír CHALUPECKÝ \& Adrian MUNTEAN

Semi-discrete finite difference multiscale scheme for a concrete corrosion model: approximation estimates and convergence

MI2011-14 Jun-ichi INOGUCHI, Kenji KAJIWARA, Nozomu MATSUURA \& Yasuhiro OHTA Explicit solutions to the semi-discrete modified KdV equation and motion of discrete plane curves

MI2011-15 Hiroshi INOUE

A generalization of restricted isometry property and applications to compressed sensing

MI2011-16 Yu KAWAKAMI

A ramification theorem for the ratio of canonical forms of flat surfaces in hyperbolic three-space

MI2011-17 Naoyuki KAMIYAMA

Matroid intersection with priority constraints

MI2012-1 Kazufumi KIMOTO \& Masato WAKAYAMA

Spectrum of non-commutative harmonic oscillators and residual modular forms

MI2012-2 Hiroki MASUDA

Mighty convergence of the Gaussian quasi-likelihood random fields for ergodic Levy driven SDE observed at high frequency 
MI2012-3 Hiroshi INOUE

A Weak RIP of theory of compressed sensing and LASSO

MI2012-4 Yasuhide FUKUMOTO \& Youich MIE

Hamiltonian bifurcation theory for a rotating flow subject to elliptic straining field

MI2012-5 Yu KAWAKAMI

On the maximal number of exceptional values of Gauss maps for various classes of surfaces

MI2012-6 Marcio GAMEIRO, Yasuaki HIRAOKA, Shunsuke IZUMI, Miroslav KRAMAR, Konstantin MISCHAIKOW \& Vidit NANDA

Topological Measurement of Protein Compressibility via Persistence Diagrams

MI2012-7 Nobutaka NAKAZONO \& Seiji NISHIOKA

Solutions to a $q$-analog of Painlevé III equation of type $D_{7}^{(1)}$

MI2012-8 Naoyuki KAMIYAMA

A new approach to the Pareto stable matching problem

MI2012-9 Jan BREZINA \& Yoshiyuki KAGEI

Spectral properties of the linearized compressible Navier-Stokes equation around time-periodic parallel flow

MI2012-10 Jan BREZINA

Asymptotic behavior of solutions to the compressible Navier-Stokes equation around a time-periodic parallel flow

MI2012-11 Daeju KIM, Shuichi KAWANO \& Yoshiyuki NINOMIYA

Adaptive basis expansion via the extended fused lasso

MI2012-12 Masato WAKAYAMA

On simplicity of the lowest eigenvalue of non-commutative harmonic oscillators

MI2012-13 Masatoshi OKITA

On the convergence rates for the compressible

Navier- Stokes equations with potential force

MI2013-1 Abuduwaili PAERHATI \& Yasuhide FUKUMOTO

A Counter-example to Thomson-Tait-Chetayev's Theorem

MI2013-2 Yasuhide FUKUMOTO \& Hirofumi SAKUMA

A unified view of topological invariants of barotropic and baroclinic fluids and their application to formal stability analysis of three-dimensional ideal gas flows

MI2013-3 Hiroki MASUDA

Asymptotics for functionals of self-normalized residuals of discretely observed stochastic processes

MI2013-4 Naoyuki KAMIYAMA

On Counting Output Patterns of Logic Circuits

MI2013-5 Hiroshi INOUE

RIPless Theory for Compressed Sensing 
MI2013-6 Hiroshi INOUE

Improved bounds on Restricted isometry for compressed sensing

MI2013-7 Hidetoshi MATSUI

Variable and boundary selection for functional data via multiclass logistic regression modeling

MI2013-8 Hidetoshi MATSUI

Variable selection for varying coefficient models with the sparse regularization

MI2013-9 Naoyuki KAMIYAMA

Packing Arborescences in Acyclic Temporal Networks

MI2013-10 Masato WAKAYAMA

Equivalence between the eigenvalue problem of non-commutative harmonic oscillators and existence of holomorphic solutions of Heun's differential equations, eigenstates degeneration, and Rabi's model

MI2013-11 Masatoshi OKITA

Optimal decay rate for strong solutions in critical spaces to the compressible Navier-

Stokes equations

MI2013-12 Shuichi KAWANO, Ibuki HOSHINA, Kazuki MATSUDA \& Sadanori KONISHI

Predictive model selection criteria for Bayesian lasso

MI2013-13 Hayato CHIBA

The First Painleve Equation on the Weighted Projective Space

MI2013-14 Hidetoshi MATSUI

Variable selection for functional linear models with functional predictors and a functional response

MI2013-15 Naoyuki KAMIYAMA

The Fault-Tolerant Facility Location Problem with Submodular Penalties

MI2013-16 Hidetoshi MATSUI

Selection of classification boundaries using the logistic regression

MI2014-1 Naoyuki KAMIYAMA

Popular Matchings under Matroid Constraints

MI2014-2 Yasuhide FUKUMOTO \& Youichi MIE

Lagrangian approach to weakly nonlinear interaction of Kelvin waves and a symmetrybreaking bifurcation of a rotating flow

MI2014-3 Reika AOYAMA

Decay estimates on solutions of the linearized compressible Navier-Stokes equation around a Parallel flow in a cylindrical domain

MI2014-4 Naoyuki KAMIYAMA

The Popular Condensation Problem under Matroid Constraints 
MI2014-5 Yoshiyuki KAGEI \& Kazuyuki TSUDA

Existence and stability of time periodic solution to the compressible Navier-Stokes equation for time periodic external force with symmetry

MI2014-6 This paper was withdrawn by the authors.

MI2014-7 Masatoshi OKITA

On decay estimate of strong solutions in critical spaces for the compressible NavierStokes equations

\section{MI2014-8 Rong ZOU \& Yasuhide FUKUMOTO}

Local stability analysis of azimuthal magnetorotational instability of ideal MHD flows

MI2014-9 Yoshiyuki KAGEI \& Naoki MAKIO

Spectral properties of the linearized semigroup of the compressible Navier-Stokes equation on a periodic layer

MI2014-10 Kazuyuki TSUDA

On the existence and stability of time periodic solution to the compressible NavierStokes equation on the whole space

MI2014-11 Yoshiyuki KAGEI \& Takaaki NISHIDA

Instability of plane Poiseuille flow in viscous compressible gas

MI2014-12 Chien-Chung HUANG, Naonori KAKIMURA \& Naoyuki KAMIYAMA

Exact and approximation algorithms for weighted matroid intersection

MI2014-13 Yusuke SHIMIZU

Moment convergence of regularized least-squares estimator for linear regression model

MI2015-1 Hidetoshi MATSUI

Sparse regularization for multivariate linear models for functional data

MI2015-2 Reika AOYAMA \& Yoshiyuki KAGEI

Spectral properties of the semigroup for the linearized compressible Navier-Stokes equation around a parallel flow in a cylindrical domain

MI2015-3 Naoyuki KAMIYAMA

Stable Matchings with Ties, Master Preference Lists, and Matroid Constraints

MI2015-4 Reika AOYAMA \& Yoshiyuki KAGEI

Large time behavior of solutions to the compressible Navier-Stokes equations around a parallel flow in a cylindrical domain

MI2015-5 Kazuyuki TSUDA

Existence and stability of time periodic solution to the compressible Navier-StokesKorteweg system on $R^{3}$

MI2015-6 Naoyuki KAMIYAMA

Popular Matchings with Ties and Matroid Constraints 